\title{
Entorhinal Stellate Cells Show Preferred Spike Phase-Locking to Theta Inputs That Is Enhanced by Correlations in Synaptic Activity
}

\author{
Fernando R. Fernandez, ${ }^{1}$ Paola Malerba, ${ }^{1}$ Paul C. Bressloff, ${ }^{2}$ and John A. White ${ }^{1}$ \\ ${ }^{1}$ Department of Bioengineering, Brain Institute, University of Utah, Salt Lake City, Utah, 84112 and ${ }^{2}$ Department of Mathematics, University of Utah, Salt \\ Lake City, Utah 84132
}

In active networks, excitatory and inhibitory synaptic inputs generate membrane voltage fluctuations that drive spike activity in a probabilistic manner. Despite this, some cells in vivo show a strong propensity to precisely lock to the local field potential and maintain a specific spike-phase relationship relative to other cells. In recordings from rat medial entorhinal cortical stellate cells, we measured spike phase-locking in response to sinusoidal "test" inputs in the presence of different forms of background membrane voltage fluctuations, generated via dynamic clamp. We find that stellate cells show strong and robust spike phase-locking to theta (4-12 $\mathrm{Hz})$ inputs. This response occurs under a wide variety of background membrane voltage fluctuation conditions that include a substantial increase in overall membrane conductance. Furthermore, the $I_{H}$ current present in stellate cells is critical to the enhanced spike phase-locking response at theta. Finally, we show that correlations between inhibitory and excitatory conductance fluctuations, which can arise through feedback and feedforward inhibition, can substantially enhance the spike phase-locking response. The enhancement in locking is a result of a selective reduction in the size of low-frequency membrane voltage fluctuations due to cancellation of inhibitory and excitatory current fluctuations with correlations. Hence, our results demonstrate that stellate cells have a strong preference for spike phase-locking to theta band inputs and that the absolute magnitude of locking to theta can be modulated by the properties of background membrane voltage fluctuations.

\section{Introduction}

Numerous intracellular in vivo cortical and hippocampal recordings have established the prevalence of stochastic membrane voltage fluctuations that drive spike activity in a probabilistic manner (Paré et al., 1998; Destexhe and Paré, 1999; Harvey et al., 2009; Epsztein et al., 2010). Neuronal spike timing in vivo, however, is often characterized by a precise relationship to the local field potential that arises from coordinated population activity. This behavior is often referred to as phase coupling or spike phaselocking and it is believed to be a mark of the precise temporal relationship between different cells as wells as an indicator of temporal coding (Fox et al., 1986; Stewart et al., 1992; O'Keefe and Recce, 1993; Csicsvari et al., 1999; Fries et al., 2001; Klausberger et al., 2003; Buzsáki and Draguhn, 2004; Klausberger and Somogyi, 2008; Rutishauser et al., 2010). Accordingly, understanding the factors involved in establishing spike phaselocking in neurons requires taking into consideration the interplay between the stochastic synaptic background activity

Received Aug. 15, 2012; revised Feb. 15, 2013; accepted Feb. 22, 2013.

Author contributions: F.R.F. and P.M. designed research; F.R.F. and P.M. performed research; F.R.F., P.M., and P.C.B. analyzed data; F.R.F. and J.A.W. wrote the paper.

This work was supported with grants from the National Institutes of Health (R01MH085387 and R01MH085074). We thank Tilman Broicher and Michael N. Economo for extensive discussions and comments on this manuscript.

Correspondence should be addressed to Fernando R. Fernandez, Department of Bioengineering, University of Utah, 36 Wasatch Drive, Salt Lake City, UT 84112. E-mail: f.fernandez@utah.edu.

DOI:10.1523/JNEUROSCI.3892-12.2013

Copyright $\odot 2013$ the authors $\quad 0270-6474 / 13 / 336027-14 \$ 15.00 / 0$ arising from network activity as well as the intrinsic membrane properties of neurons.

Like many cells in the hippocampal region, layer II stellate cells of medial entorhinal cortex (MEC) lock strongly to the local theta $(4-12 \mathrm{~Hz})$ field potential while receiving stochastic fluctuating inputs in vivo (Alonso and García-Austt, 1987; Stewart et al., 1992; Frank et al., 2001; Quilichini et al., 2010; Brandon et al., 2011). Stellate cell phase-locking, and thus temporal coding, are hypothesized to be driven by intrinsic properties (Alonso and Llinás, 1989; Haas and White, 2002). Several groups (O'Keefe and Burgess, 2005; Burgess et al., 2007; Giocomo et al., 2007; Brandon et al., 2011; Koenig et al., 2011) have hypothesized further that intrinsically driven stellate cell phase-locking contributes to the grid-like receptive fields of MEC cells (Hafting et al., 2005). For these reasons, the mechanisms of stellate cells phase-locking are of considerable general interest.

In our previous work we established that stellate cells, when driven by fluctuating, conductance-based inputs, fail to generate autonomous theta oscillations (Fernandez and White, 2008), seemingly in conflict with the above-cited results. However, a potential frequency preference at theta frequencies that leads to greater spike phase-locking to theta band inputs has not been studied. Thus, while stellate cells may not generate autonomous oscillations they may still support an amplification of theta inputs via an intrinsic preference for these inputs.

To understand the relationship between synaptic activity, membrane voltage fluctuations, and spike timing, we recorded 
from stellate cells in acute rat brain slices. We used dynamic clamp to simulate different types of background activity taking into account the possible conductance and correlation properties of excitatory and inhibitory synaptic activity present in vivo, and probed spike phase-locking in response to sinusoidal test inputs. We find that intrinsic cellular mechanisms endow stellate cells with a strong and robust preference for locking to theta inputs under a variety of conditions that include both low and high conductance states. Nevertheless, the magnitude of spike phaselocking can be modulated by the statistical properties of background synaptic activity.

\section{Materials and Methods}

Tissue preparation. All experimental protocols were approved by the University of Utah Institutional Animal Care and Use Committee. Horizontal sections of hippocampus and entorhinal cortex were prepared from 22- to 35-d-old Long-Evans rats of either sex. All chemicals were obtained from Sigma-Aldrich unless otherwise noted. After anesthetization with isoflurane and decapitation, brains were removed and immersed in $0^{\circ} \mathrm{C}$ solution consisting of the following (in mM): 215 sucrose, 25 $\mathrm{NaHCO}_{3}, 20$ D-glucose, $2.5 \mathrm{KCl}, 0.5 \mathrm{CaCl}_{2}, 125 \mathrm{NaH}_{2} \mathrm{PO}_{4}$, and $3 \mathrm{MgCl}_{2}$, buffered to $\mathrm{pH} 7.4$ with $95 / 5 \% \mathrm{O}_{2} / \mathrm{CO}_{2}$. Horizontal slices were cut to a thickness of $400 \mu \mathrm{m}$ (Leica VT 1200; Leica Microsystems). After the cutting procedure, slices were incubated in artificial CSF (ACSF) at $30^{\circ} \mathrm{C}$ for $20 \mathrm{~min}$ before being cooled to room temperature $\left(20^{\circ} \mathrm{C}\right)$. The ACSF consisted of the following (in mM): $125 \mathrm{NaCl}, 25 \mathrm{NaHCO}_{3}, 25 \mathrm{D}$-glucose, $2 \mathrm{KCl}, 2 \mathrm{CaCl}_{2}, 1.25 \mathrm{NaH}_{2} \mathrm{PO}_{4}$, and $1 \mathrm{MgCl}_{2}$, and buffered to $\mathrm{pH} 7.4$ with $95 / 5 \% \mathrm{O}_{2} / \mathrm{CO}_{2}$. After the incubation period, slices were moved to the stage of an infrared differential interference contrast-equipped microscope (Axioscop 2+; Zeiss). On occasion, ACSF containing $10 \mu \mathrm{M}$ CNQX, $50 \mu \mathrm{m}$ picrotoxin, and $30 \mu \mathrm{M}$ AP-5 was used to block ionotropic synaptic activity and test the effect of background activity on membrane resistance. We did not observe any significant effect on membrane resistance with application of these drugs. All recordings were conducted between 32 and $34^{\circ} \mathrm{C}$.

Electrophysiology. Electrodes were drawn on a horizontal puller (P97; Sutter Instruments) and filled with an intracellular solution consisting of the following (in mM): $120 \mathrm{~K}$-gluconate, $20 \mathrm{KCl}, 10$ HEPES, 7 diTrisPhCr, $4 \mathrm{Na}_{2} \mathrm{ATP}, 2 \mathrm{MgCl}_{2}, 0.3$ Tris-GTP, and 0.2 EGTA, and buffered to $\mathrm{pH} 7.3$ with $\mathrm{KOH}$. Final electrode resistances were between 3 and $4 \mathrm{M} \Omega$, with access resistance values between 4 and $12 \mathrm{M} \Omega$. Electrophysiological recordings were performed with a current-clamp amplifier (Axoclamp 2B; Molecular Devices), and data were acquired using custom software developed in MATLAB (v. 2011; MathWorks) using the data acquisition toolbox.

For dynamic clamp experiments, the current-clamp amplifier was driven by an analog signal from an $\times 86$ personal computer running Real-Time Application Interface Linux and an updated version of the Real-Time Linux Dynamic Clamp called Real-Time eXperimental Interface (Bettencourt et al., 2008). For all experiments, a noisy synaptic stimulus was generated using two independent conductances representing synaptic inhibition $\left(g_{i}\right)$ and excitation $\left(g_{e}\right)$ as follows:

$$
I_{\text {noise }}=g_{i}(t)\left(V-E_{i}\right)+g_{e}(t)\left(V-E_{e}\right) .
$$

The reversal potentials for inhibition $\left(E_{i}\right)$ and excitation $\left(E_{e}\right)$ were set to -75 and $0 \mathrm{mV}$, respectively. As in previous studies (Destexhe et al., 2001; Fellous et al., 2003), the terms $g_{i}(t)$ and $g_{e}(t)$ were implemented using Ornstein-Uhlenbeck (OU) processes and modeled using the following equations:

$$
\begin{aligned}
& \frac{d g_{i}}{d t}=\frac{-\left(g_{i}-\bar{g}_{i}\right)}{\tau_{i}}+\sqrt{D_{i}} \chi_{i}(t), \\
& \frac{d g_{e}}{d t}=\frac{-\left(g_{e}-\bar{g}_{e}\right)}{\tau_{e}}+\sqrt{D_{e}} \chi_{e}(t) .
\end{aligned}
$$

In the above equations, $\bar{g}_{i}$ and $\bar{g}_{e}$ represent the mean conductance for inhibition and excitation. $D_{i}$ and $D_{e}$ represent the diffusion coefficients and $\chi_{i}$ and $\chi_{e}$ are Gaussian white noise terms with unit standard deviation (SD). The terms $\tau_{i}$ and $\tau_{e}$ correspond to the time constant for inhibition and excitation, which were set to 8 and $2 \mathrm{~ms}$, respectively. In addition, $D_{i}$ and $D_{e}$ were set so that SD ratio between $g_{i}$ and $g_{e}$ fluctuations $(\rho)$ of 4,2 , or 1 were generated. A SD of $\sim 1.5 \mathrm{mV}$ was used for membrane voltage output.

The correlation between inhibition and excitation was manipulated by introducing correlation in the random Gaussian processes controlling inhibition and excitation. Correlations were set using the following equation:

$$
\chi_{i}=c \chi_{e}+\sqrt{1-c^{2}} \chi_{r} .
$$

In the above, $\chi_{i}$ and $\chi_{e}$ are Gaussian random variables associated with inhibition and excitation, while $\chi_{r}$ is independent of $\chi_{e}$. The term $c$ is the correlation coefficient relating the correlation magnitude between $\chi_{i}$ and $\chi_{e}$

For experiments with fluctuations and added conductance, $\bar{g}_{i}$ and $\bar{g}_{e}$ were kept at a 4:1 ratio and set to 12 and $3 \mathrm{nS}$, respectively, so that mean current amplitudes would be approximately balanced. In experiments where $\bar{g}_{i}$ and $\bar{g}_{e}$ were set to 12 and $3 \mathrm{nS}$ (high conductance), $g_{i}$ and $g_{e}$ were not rectified. This was not an issue as the mean values for $g_{i}$ and $g_{e}$ were sufficiently far from zero that fluctuations did not cross zero and become negative. For conditions where fluctuations were added without a mean conductance component $\left(\bar{g}_{i}\right.$ and $\bar{g}_{e}=0$, low conductance), conductances were rectified so that a negative conductance was always avoided. Under these conditions, a small mean positive conductance $(\sim 1 \mathrm{nS})$ was introduced at some time points by the rectified $g_{i}$ and $g_{e}$ fluctuations.

In cases where conductance was added without membrane voltage fluctuations, a simple linear leak conductance was introduced via dynamic clamp using the equation:

$$
I_{\text {leak }}=g_{\text {leak }}\left(V-E_{\text {leak }}\right) .
$$

For these experiments, $E_{\text {leak }}$ was set to $-75 \mathrm{mV}$. For all experiments, the sample rate of the dynamic clamp was set to $10 \mathrm{kHz}$. A measured junction potential of $\sim 10 \mathrm{mV}$ was subtracted from all recordings and taken into account during dynamic clamp experiments. Data were collected between 5 and $20 \mathrm{kHz}$ and filtered at $3 \mathrm{kHz}$.

Analysis and statistics. All analyses were performed in MATLAB using custom software and/or built in functions. Spike times were determined using a threshold crossing for membrane voltage. Spike phase-locking index (vector strength) is defined as summed normalized vector length of circular data and provides a measure of phase-locking of a series of spiking events relative to an ongoing oscillation. Spike phase-locking index was calculated using the following equation:

$$
p=\frac{1}{n} \sum_{j=1}^{n} e^{i \omega t_{i}}, \omega=\frac{2 \pi}{T}
$$

where $T=$ period of modulation frequency, $t_{j}=$ spike times. Each $e^{i \omega t_{i}}$ is then a unit length vector representing the phase of the spike. Phaselocking index was defined as the length (modulus) of the vector $p$ (see Fig. $1 B)$. For each measure of phase-locking index at a given modulation frequency, the total number of spikes $(n)$ was always $>1700$ (700 s recordings at an average firing rate of $\sim 2.5$ spikes/s). Modulation frequencies of 1, 2, 5, 8, 12, 15, and $30 \mathrm{~Hz}$ were used and applied via a small (20 pA peak-to-peak) sinusoidal current injection in the presence of membrane voltage fluctuations.

Power spectra were calculated using MATLAB's $p s d$ or $p w e l c h$ functions. For experiments using filtered current input noise, we constructed a white noise process in the frequency domain and applied the following first order filters to generate low-pass or bandpass current noise:

$$
H(s)=\frac{1}{1+s \tau} \text { (low-pass), } H(s)=\frac{s \tau}{1+s \tau} \text { (high-pass). }
$$

The low-pass ( $\tau=0.01 \mathrm{~s}$ ) filter was used to construct current input noise with a spectra similar to current input delivered during uncor- 
related synaptic input. The combination of low- and high-pass $(\tau=$ $0.03 \mathrm{~s}$ ) filters was used to generate a bandpass current input with a spectra similar to that observed using certain correlated inputs (see Fig. 7A). MATLAB's ifft function was used to implement an inverse Fourier transform and generate the time series from signals constructed in the frequency domain.

The current protocol for input impedance measurements consisted of a Gaussian current input that was filtered at $100 \mathrm{~Hz}$ (same as the low-pass filtered signal above). Impedance $(Z(f))$ measures were calculated by taking the ratio of the fast Fourier transform of the membrane voltage response $(V)$ and current input $(I)$ stimulus:

$$
Z(f)=\frac{|F F T(V)|}{|F F T(I)|} .
$$

For multiple comparisons, statistical significance was determined using either a one- or two-way ANOVA. For repeated measures of means, statistical difference was determined using Tukey's honestly significant criteria. A $t$ test (one- or two-sample) was used when comparing one or two means. Means are presented along with the SEM.

Mathematical results. In this section we derive the mathematical relationship describing how correlated synaptic inputs shape the power spectra of synaptic inputs. This relationship allowed us to quickly and rigorously determine the major factors that shape synaptic power spectra (see Fig. 4), making predictions that suggested specific methods of data analysis (see Figs. 5, 6), as well as follow-up experiments (Fig. 7) that strongly tested the theoretical predictions. Consider a passive neural membrane receiving the same inputs used in our experiments:

$$
\left\{\begin{array}{l}
C \dot{V}=-g_{L}\left(V-V_{L}\right)-g_{e}(t)\left(V-E_{e}\right)-g_{i}(t)\left(V-E_{i}\right) \\
\dot{g}_{e}=-\frac{g_{e}-\bar{g}_{e}}{\tau_{e}}+\sqrt{D_{e}} \chi_{e}(t) \\
\dot{g}_{i}=-\frac{g_{i}-\bar{g}_{i}}{\tau_{i}}+\sqrt{D_{i}} \chi_{i}(t)
\end{array}\right.
$$

where $\chi_{x}$ are white noises for $x=e, i$ and $\chi_{i}(t) \stackrel{\text { def }}{=}$ $c \chi_{e}(t)+\sqrt{1-c^{2}} \chi(t)$, for $0<c<1$, consistent with Equation 3 .

Also, in this section we refer to the following parameter combination: $C=1 \mu \mathrm{F} / \mathrm{cm}^{2}, E_{L}=-60 \mathrm{mV}, g_{L}=0.1 \mathrm{mS} / \mathrm{cm}^{2}, E_{e}=0 \mathrm{mV}, \bar{g}_{e}$ $=0.05 \mathrm{mS} / \mathrm{cm}^{2}, E_{i}=-75 \mathrm{mV}, \bar{g}_{i}=0.2 \mathrm{mS} / \mathrm{cm}^{2}$, and $0 \leq c \leq 1$. In the experiments $\rho=\sigma_{i} / \sigma_{e}$ is assumed constant, where $\sigma_{x}$ for $x=c, i$ are the SDs of the excitatory and inhibitory input conductance noises, respectively. To replicate such condition on $\rho$, we recall that the stationary SD of each OU process is well known $\sigma_{x}=\sqrt{D_{x} \tau_{x} / 2}$ (for $x=e, i)$, so we assume the following:

$$
D_{i}{ }^{d e f} \rho^{2} D_{e} \frac{\tau_{e}}{\tau_{i}} \text { for } \rho \in \mathbb{N},
$$

where $D_{c}=0.0001$.

We can consider the input current

$$
I(t) \stackrel{\text { def }}{=} g_{e}(t)\left(V-E_{e}\right)+g_{i}(t)\left(V-E_{i}\right),
$$

and look for the power spectrum of $I(t)$ (referred to as total synaptic input current; see Results), which we label $P S_{I}$. In particular, we are interested in assessing the role of the parameters $c, \rho, \tau_{e}$, and $\tau_{i}$ in the monotonicity of $P S_{I}$. We first derive an approximated form for $I(t)$, and then use the Weiner-Khinchine theorem (Pécseli, 2000), which guarantees that we can compute $P S_{I}$ as the Fourier transform of the autocorrelation function of $I(t)$.

To find $I(t)$, we need to derive an approximated solution to System 8 . The multiplicative role of noise in the first equation makes the derivation of a closed analytic solution impossible, hence we will use a voltage estimate in Equation 10. We first perform an Effective Time Constant Approximation (Richardson and Gerstner, 2005) as follows:

$$
\left\{\begin{array}{l}
C \dot{V}=g_{0}\left(V-E_{0}\right)-\tilde{g}_{e}(t)\left(E_{0}-E_{e}\right)-\tilde{g}_{i}(t)\left(E_{0}-E_{i}\right) \\
\dot{\tilde{g}}_{e}=-\frac{\tilde{g}_{e}}{\tau_{e}}+\sqrt{D_{e}} \chi_{e}(t) \\
\dot{\tilde{g}}_{i}=-\frac{\tilde{g}_{i}}{\tau_{i}}+\sqrt{D_{i}} \chi_{i}(t)
\end{array}\right.
$$

where $g_{0}=g_{L}+\bar{g}_{e}+\bar{g}_{i}$ and $E_{0}=\left(g_{L} E_{L}+\bar{g}_{e} E_{e}+\bar{g}_{i} E_{i}\right) / g_{0}$. We label $\tilde{g}_{x}(t)=g_{x}(t)-\bar{g}_{x}$ for $x=e, i$. In fact, Richardson and Gerstner (2005) have shown that the main Gaussian properties of the voltage distribution are captured in this approximation, which is the leading order solution to the full conductance-based model in the presence of synaptic shot noise. To further simplify our derivation, we consider the following:

$$
\tilde{g}_{x}(t) \approx \sigma_{x} \chi_{x}(t) \text { for } x=e, i
$$

treating each conductance as a white noise process. This approximation can be chosen in additive noise problems, though it treats the autocorrelation of each noise process (excitatory and inhibitory) as a delta function. Then the first equation in System 11 becomes the following:

$$
\dot{V}=-g_{0}\left(V-E_{0}\right)-\sigma_{e}\left(E_{0}-E_{e}\right) \chi_{e}-\sigma_{i}\left(E_{0}-E_{i}\right) \chi_{i},
$$

which suggest to consider

$$
\begin{gathered}
\tilde{\sigma}_{e}{ }^{d e f} \sigma_{e}\left(E_{0}-E_{e}\right), \\
\tilde{\sigma}_{i}^{d e f} \sigma_{i}\left(E_{0}-E_{i}\right),
\end{gathered}
$$

as $\tilde{\sigma}_{e}$ and $\tilde{\sigma}_{i}$ are the SDs of the white noises used in Equation 13. Note that the parameter $\rho$ acquires a specific role in this redefined problem. In fact, if we consider the ratio (in absolute value) of the new SDs as follows:

$$
\left|\frac{\tilde{\sigma}_{i}}{\tilde{\sigma}_{e}}\right|=\rho\left|\frac{E_{0}-E_{i}}{E_{0}-E_{e}}\right|,
$$

we can see how in physiological ranges of the reversal potentials the ratio $\left(E_{0}-E_{i}\right) /\left(E_{0}-E_{e}\right)$ controls the range of values of $\rho$, so that the conductance noises will have comparable variations.

Equation 13 can now be solved with standard Itō calculus:

$$
\begin{array}{r}
V(t)=e^{-g_{0} t}\left(V_{0}-E_{0}\right)+E_{0}+\tilde{\sigma}_{i} \int_{0}^{t} e^{-g_{0}(t-s)} d B_{s}^{i} \\
+\tilde{\sigma}_{e} \int_{0}^{t} e^{-g_{0}(t-s)} d B_{s}^{e},
\end{array}
$$

where $\chi_{x}(t) d t=d B_{t}^{x}$ for $x=e, i$. The equations in System 8 defining the OU processes give the following:

$$
g_{e}(t)=e^{-t / \tau_{e}}\left(g_{e}(0)-\bar{g}_{e}\right)+\bar{g}_{e}+\sqrt{D_{e}} \int_{0}^{t} e^{-(t-s) / \tau_{e}} d B_{s}^{e},
$$

$$
g_{i}(t)=e^{-t / \tau_{i}}\left(g_{i}(0)-\bar{g}_{i}\right)+\bar{g}_{i}+\sqrt{D_{i}} \int_{0}^{t} e^{-(t-s) / \tau_{i}} d B_{s}^{i},
$$


(The assumptions $g_{x}>0$ for $x=e, i$, necessary to the above derivation, are satisfied throughout the experimental conditions). We can therefore merge Equations 16-18 in Equation 10 to obtain our approximated $I(t)$ stochastic process.

To compute $P S_{I}(f)$, we need the autocorrelation of $I(t)$, which we calculate as follows:

$$
A c f_{I(t)}(\tau)=E[(I(t)-\overline{I(t)})(I(t+\tau)-\overline{I(t+\tau)})] .
$$

Note that the expectation is taken with respect to trials, i.e., over noise, and that because of causality (our experiments have an initial time, $t=$ 0 ), all our calculations have been performed in positive time.

We find that the stationary autocorrelation of the synaptic current in System 11 is given by the linear combination of three decaying exponentials, one per timescale in the system:

$$
A c f_{I(\infty)} \tau=F_{g_{0}} e^{-g_{0} \tau}+F_{\tau_{e}} e^{-\tau / \tau_{e}}+F_{\tau_{i}} e^{-\tau / \tau_{i}}
$$

where

$$
\begin{aligned}
F_{g_{0}=}^{\operatorname{def}} \frac{1}{2 g_{0}}\left(\bar{g}_{e}+\bar{g}_{i}\right)^{2}\left(\tilde{\sigma}_{i}^{2}+\tilde{\sigma}_{e}^{2}+2 c \tilde{\sigma}_{i} \sigma_{e}\right) & \\
+ & \frac{\sqrt{2 \tau_{e}}}{1+g_{0} \tau_{e}}\left(\bar{g}_{e}+\bar{g}_{i}\right) \tilde{\sigma}_{e}\left(c \tilde{\sigma}_{i}+\tilde{\sigma}_{e}\right) \\
& +\frac{\sqrt{2 \tau_{i}}}{1+g_{0} \tau_{i}}\left(\bar{g}_{e}+\bar{g}_{i}\right) \tilde{\sigma}_{i}\left(\tilde{\sigma}_{i}+c \tilde{\sigma}_{e}\right)
\end{aligned}
$$

$F_{\tau_{e}=}^{d e f} \frac{\sqrt{2 \tau_{e}}}{1+g_{0} \tau_{e}}\left(\bar{g}_{e}+\bar{g}_{i}\right) \tilde{\sigma}_{e}\left(c \tilde{\sigma}_{i}+\tilde{\sigma}_{e}\right)+\tilde{\sigma}_{e}^{2}$

$$
+2 \frac{\sqrt{\tau_{e} \tau_{i}}}{\tau_{i}+\tau_{e}} c \tilde{\sigma}_{i} \tilde{\sigma}_{e}
$$

and

$$
\begin{aligned}
F_{\tau_{i}}{ }^{\operatorname{def}} \frac{\sqrt{2 \tau_{i}}}{1+g_{0} \tau_{i}}\left(\bar{g}_{e}+\bar{g}_{i}\right) \tilde{\sigma}_{i}\left(\tilde{\sigma}_{i}+c \tilde{\sigma}_{e}\right) & +\tilde{\sigma}_{i}^{2} \\
& +2 \frac{\sqrt{\tau_{e} \tau_{i}}}{\tau_{i}+\tau_{e}} c \tilde{\sigma}_{i} \tilde{\sigma}_{e} .
\end{aligned}
$$

Now $P S_{I}$ is the squared modulus of the Fourier transform of Equation 20. The transform is given by the following:

$$
\begin{aligned}
A c \widehat{f_{I(\infty)}}(\tau)(f) & =\left(F_{g_{0}} e^{-g_{0} \tau}+F_{\tau_{e}} e^{-\tau / \tau_{e}}+F_{\tau_{i}} e^{-\widehat{\tau} / \tau_{i}}\right)(f) \\
& =\frac{F_{g_{0}}}{g_{0}+2 \pi i f}+\frac{F_{\tau_{e}}}{\tau_{e}^{-1}+2 \pi i f}+\frac{F_{\tau_{i}}}{\tau_{i}^{-1}+2 \pi i f},
\end{aligned}
$$

and the relative sizes and signs of the linear factors $F_{g_{0}}, F_{\tau_{e}}$, and $F_{\tau_{i}}$ will establish if the power spectrum of $I(t)$ is monotonic or not.

The squared modulus of this complex function at every $f$ value is the sum of the square of its real and imaginary part:

$$
\begin{gathered}
P S_{I}(f)=\left\|A c \widehat{f_{I(\infty)}}(f)\right\|^{2} \\
=\left[\frac{g_{0} F_{g_{0}}}{g_{0}^{2}+(2 \pi f)^{2}}+\frac{\tau_{e}^{-1} F_{\tau_{e}}}{\tau_{e}^{-2}+(2 \pi f)^{2}}+\frac{\tau_{i}^{-1} F_{\tau_{i}}}{\tau_{i}^{-2}+(2 \pi f)^{2}}\right]^{2} \\
+\left[( - 2 \pi f ) \left(\frac{F_{g_{0}}}{g_{0}^{2}+(2 \pi f)^{2}}+\frac{F_{\tau_{e}}}{\tau_{e}^{-2}+(2 \pi f)^{2}}\right.\right. \\
\left.\left.+\frac{F_{\tau_{i}}}{\tau_{i}^{-2}+(2 \pi f)^{2}}\right)\right]^{2},
\end{gathered}
$$

which provides the analytical expression for $P S_{I}$ we were looking for.
We can now explore the parameter space $\left(c, \rho, \tau_{e}, \tau_{i}\right) \in[0,1] \times[0$, $+\infty)^{3} \subset \mathbb{R}^{4}$, with respect to the monotonicity in the power spectrum of $I(t)$. To do that, we define a function $\mathcal{M}: \mathbb{R}^{4} \rightarrow \mathbb{R}^{+}$that is zero whenever $P S_{I}$ is monotonic, and is the ratio between the peak value of $P S_{I}$ and its initial value when it is non-monotonic. For any given $\left(c, \rho, \tau_{e}, \tau_{i}\right)$, let $m=\max _{f \in[0,+\infty]}\left(P S_{I}(f)\right)$ and $f^{\star}$ the frequency at which such maximum is found. Then

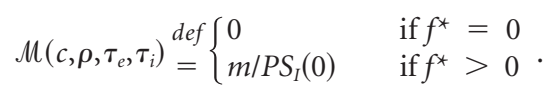

Examples of the resulting $\mathcal{M}$ values in subspaces are reported in Figure 4.

We can also compute the power spectrum for the excitatory and inhibitory synaptic current, separately:

$$
\begin{aligned}
& I_{e}(t)=g_{e}(t)\left(V-E_{e}\right), \\
& I_{i}(t)=g_{i}(t)\left(V-E_{i}\right) .
\end{aligned}
$$

Using the same procedure as for $I(t)$, we get the following:

$$
\begin{aligned}
P S_{I_{e}}(f)= & \left(\frac{g_{0} K_{g_{0}}}{g_{0}^{2}+(2 \pi f)^{2}}+\frac{\tau_{e}^{-1} K_{\tau_{e}}}{\tau_{e}^{-2}+(2 \pi f)^{2}}\right)^{2} \\
& +(2 \pi f)^{2}\left(\frac{K_{g_{0}}}{g_{0}^{2}+(2 \pi f)^{2}}+\frac{K_{\tau_{e}}}{\tau_{e}^{-2}+(2 \pi f)^{2}}\right)^{2},
\end{aligned}
$$

where

$$
\begin{gathered}
K g_{0}=\frac{\bar{g}_{e}^{2}}{2 g_{0}}\left(\tilde{\sigma}_{i}^{2}+\tilde{\sigma}_{e}^{2}+2 c \tilde{\sigma}_{i} \tilde{\sigma}_{e}\right) \\
+\bar{g}_{e} \frac{\sqrt{2 \tau_{e}}}{1+g_{0} \tau_{e}} \tilde{\sigma}_{e}\left(c \tilde{\sigma}_{i}+\tilde{\sigma}_{e}\right), \\
K_{\tau_{e}}=\tilde{\sigma}_{e}^{2}+\bar{g}_{e} \frac{\sqrt{2 \tau_{e}}}{1+g_{0} \tau_{e}} \tilde{\sigma}_{e}\left(c \tilde{\sigma}_{i}+\tilde{\sigma}_{e}\right) .
\end{gathered}
$$

Analogously, in the inhibitory current we get the following:

$$
\begin{aligned}
P S_{I_{i}}(f)= & \left(\frac{g_{0} J_{g_{0}}}{g_{0}^{2}+(2 \pi f)^{2}}+\frac{\tau_{i}^{-1} J_{\tau_{i}}}{\tau_{i}^{-2}+(2 \pi f)^{2}}\right)^{2} \\
& +(2 \pi f)^{2}\left(\frac{J_{g_{0}}}{g_{0}^{2}+(2 \pi f)^{2}}+\frac{J_{\tau_{i}}}{\tau_{i}^{-2}+(2 \pi f)^{2}}\right)^{2},
\end{aligned}
$$

where

$$
J_{g_{0}}=\frac{\bar{g}_{i}^{2}}{2 g_{0}}\left(\tilde{\sigma}_{i}^{2}+\tilde{\sigma}_{e}^{2}+2 c \tilde{\sigma}_{i} \tilde{\sigma}_{e}\right)+\bar{g}_{i} \frac{\sqrt{2 \tau_{i}}}{1+g_{0} \tau_{i}} \tilde{\sigma}_{i}\left(\tilde{\sigma}_{i}+c \tilde{\sigma}_{e}\right),
$$

$$
J_{\tau_{i}}=\tilde{\sigma}_{i}^{2}+\bar{g}_{i} \frac{\sqrt{2 \tau_{i}}}{1+g_{0} \tau_{i}} \tilde{\sigma}_{e}\left(\tilde{\sigma}_{i}+c \tilde{\sigma}_{e}\right) .
$$

Equations (29) and (32) define the curves reported in Figure 4, A, B, E, and $F$.

\section{Results}

MEC stellate cells preferentially lock to theta inputs under a variety of background conditions

Our goal in this study was to understand the intrinsic and synaptic factors establishing spike phase-locking in stellate cells. Although previous work has implicated stellate cells in theta network activity (Alonso and García-Austt, 1987; Stewart et al., 1992; Frank et al., 2001; Quilichini et al., 2010; Brandon et al., 2011; Burgalossi et al., 2011; Koenig et al., 2011), the ability for stellate cell spike output to preferentially amplify or lock to theta inputs has not been studied. In addition, we were interested in 
how spike phase-locking is potentially modulated under fluctuation-driven spiking consistent with the in vivo state. To achieve these aims, we embedded stellate cells in an artificial synaptic environment constructed in dynamic clamp using OU processes. Through the use of OU processes, we could approximate in vivo membrane voltage conditions using a variety of parameters to control synaptic inputs, and also drive spikes in a probabilistic manner (Destexhe et al., 2001; Fernandez et al., 2011). Because we introduce the artificial inputs at the cell body, mimicking inputs as measured somatically in vivo, our results necessarily focus on how spiking is affected by particular patterns of somatic voltage, current, and conductance, ignoring how dendritic inputs give rise to those patterns.

To start, we tested three distinct forms of background synaptic activity consistent with a range of different background synaptic input fluctuations measured in vivo. First, we constructed a background synaptic stimulus that provided stochastic fluctuations in membrane voltage without introducing a substantial change in stellate cell membrane conductance $(\sim 1 \mathrm{nS}$, low conductance). Second, we used a stimulus that significantly increased overall membrane conductance ( $15 \mathrm{nS}$, high conductance) while preserving the SD in membrane voltage fluctuations of the low conductance condition. The total conductance was the sum of mean inhibitory and excitatory processes with values of 12 and $3 \mathrm{nS}$, respectively. Testing the role of both low and high conductance synaptic activity is important as in vivo data has indicated that voltage fluctuations may or may not be associated with a change in average input resistance (Waters and Helmchen, 2006; Cardin et al., 2008). Finally, we used a stimulus similar to the second, but with temporal correlations between inhibitory and excitatory synaptic conductance fluctuations. Recent data indicate that cortical circuits are often characterized by the presence of feedforward and feedback inhibition (Pouille and Scanziani, 2001; Heiss et al., 2008; Atallah and Scanziani, 2009; Franks et al., 2011; Isaacson and Scanziani, 2011; Suzuki and Bekkers, 2012). As a result of this connectivity, activation of cortical circuits leads to highly correlated and proportional levels of excitation and inhibition (Anderson et al., 2001; Shu et al., 2003; Wehr and Zador, 2003; Heiss et al., 2008; Okun and Lampl, 2008; Atallah and Scanziani, 2009; Adesnik and Scanziani, 2010; Franks et al., 2011). The magnitude of correlation underlying the random processes (Eq. 3; see Materials and Methods) was set initially to a value of 1 , which due to differences in the time constants between $g_{i}$ and $g_{e}$ fluctuations produced a peak correlation between the two fluctuating conductances of $\sim 0.8$ (Okun and Lampl, 2008; Adesnik and Scanziani, 2010). For all three conditions, we maintained the SD of membrane voltage fluctuations constant and used small amounts of current injection to keep cells at an average firing rate of $2.5 \mathrm{~Hz}$ (Fig. $1 A)$. The choice of mean spike rate $(\sim 2.5 \mathrm{~Hz})$ and voltage fluctuations $(\sim 1.5-2 \mathrm{mV}$ SD) was based on in vivo observations from MEC stellate cells indicating low and variable spike rates generated by random membrane voltage fluctuations (Frank et al., 2001; Quilichini et al., 2010; Burgalossi et al., 2011). For all recordings, the membrane voltage distribution (excluding spikes) was unimodal with a range spanning -75 to $-65 \mathrm{mV}$ (Fig. $1 A 1-A 3$, top) and average SD values of $2.0 \pm 0.1 \mathrm{mV}, 1.9 \pm$ $0.1 \mathrm{mV}$, and $1.8 \pm 0.04 \mathrm{mV}$ for low conductance, high conductance, and correlated conditions, respectively $(n=8-22)$.

To evaluate the ability of stellate cells to lock at different input frequencies in the presence of stochastic voltage fluctuations, we used a small amplitude (10 or 20 pA peak-to-peak for low and high conductance, respectively) sinusoidal current input that was independent of the background synaptic activity introduced via dynamic clamp. This signal was added to the total synaptic conductance input and was varied in frequency to assess the spike phase-locking response between 1 and $30 \mathrm{~Hz}$. Spike phaselocking was quantified using an index that represents the average phase preference of the spike time relative to the sinusoidal input. An index of 0 indicates no preference for the phase of the sinusoidal input and would result in a flat spike phase histogram. Conversely, an index of 1 would indicate that all spikes fired at the exact same phase of the input. This approach is very similar to previous theoretical and experimental work characterizing neuronal spike responses to time-varying inputs (Brunel et al., 2001; Fourcaud-Trocmé et al., 2003; Köndgen et al., 2008; Rutishauser et al., 2010; Wei and Wolf, 2011; Broicher et al., 2012; Kispersky et al., 2012).

As indicated in Figure 1, the spike phase-locking response in stellate cells was sensitive to the frequency of the sinusoidal test input as well as the form of background synaptic input fluctuations (Fig. $1 C ; p<0.001$, two-way ANOVA, $n=8-22$ ). Notably, for all synaptic background conditions tested, spike phaselocking in stellate cells was significantly stronger in response to sinusoidal inputs in the theta range ( 5 and $8 \mathrm{~Hz}$ vs other frequencies, $p<0.001$, one-way ANOVA, Tukey's test). Consequently, stellate cells show a strong spike resonance behavior at theta frequencies, while in the presence of stochastic membrane voltage fluctuations and probabilistic spike discharge.

To evaluate the relative strength of the spike resonance behavior we used the ratio of spike phase-locking indices at 1 and $5 \mathrm{~Hz}$. This is analogous to the "Q-value" used for subthreshold impedance and is a measure of the relative strength of phase-locking to $5 \mathrm{~Hz}$ input; therefore we called this a Q-value as well. For all three conditions, the magnitude of spike resonance at theta was strong (Q-values $>1.4$ ) but also significantly modulated by our choice of background activity. First, background synaptic fluctuations associated with an increase in membrane conductance significantly reduced the $\mathrm{Q}$-value relative to a similar background with little added conductance (Fig. $1 D ; 1.75 \pm 0.08$ vs $1.49 \pm 0.05, p=$ 0.01 , Tukey's test, $n=8-22$ ). Introducing correlations between $g_{i}$ and $g_{e}$ modestly increased the Q-value. This result, however, did not reach significance using Tukey's test (Fig. $1 D ; p=0.09$ ). In addition to a reduction in the $\mathrm{Q}$-value, increasing membrane conductance led to a significant increase in spike phase-locking to high-frequency $(8-30 \mathrm{~Hz})$ sinusoidal inputs (Fig. $1 C$; $p<0.02$, one-way ANOVA, Tukey's test, $n=8$ ), while decreasing locking to $2 \mathrm{~Hz}$ input (Fig. $1 C ; p=0.01$ one-way ANOVA, Tukey's test, $n=8$ ). Nevertheless, the spike phase-locking relationship retained a resonance profile with a peak at theta under low and high membrane conductance conditions (Fig. 1C). The largest effects on spike phase-locking, however, occurred with background synaptic activity incorporating temporal correlations between $g_{i}$ and $g_{e}$. With correlations, there was a large enhancement of spike phase-locking to most input frequencies, including the theta range (Fig. $1 C, E ; p<0.001$, one-way ANOVA, $n=22$ ). Hence, stellate cells show a strong preference for locking to theta band inputs and the overall strength of locking is significantly modulated by factors controlling the conductance and correlation properties of the background synaptic input.

Preference for theta inputs in stellate cells is established by $I_{H}$ Due to the prevalence of strong spike phase-locking to theta inputs, we hypothesized that the selectivity for theta inputs in stellate cell spike activity was related to an intrinsic property and not the specific choice of artificial background synaptic stimulus. It is well established that MEC stellate cells display a prominent sub- 
A1 low conductance
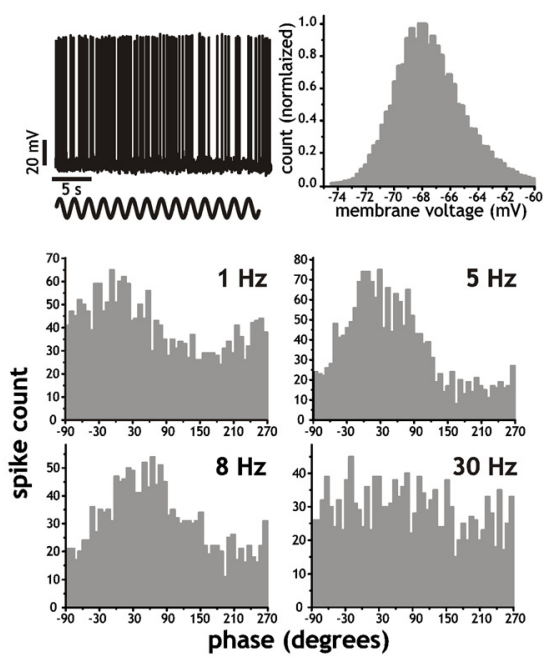

A2
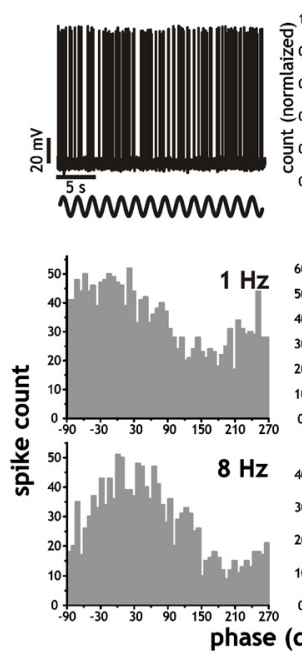

A3
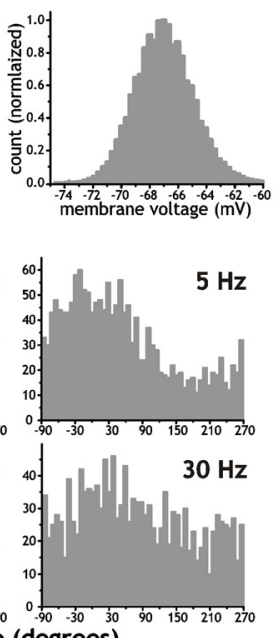

$30 \mathrm{~Hz}$

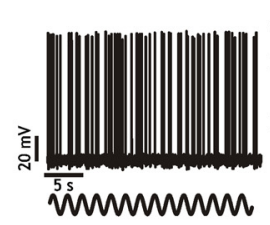

correlated
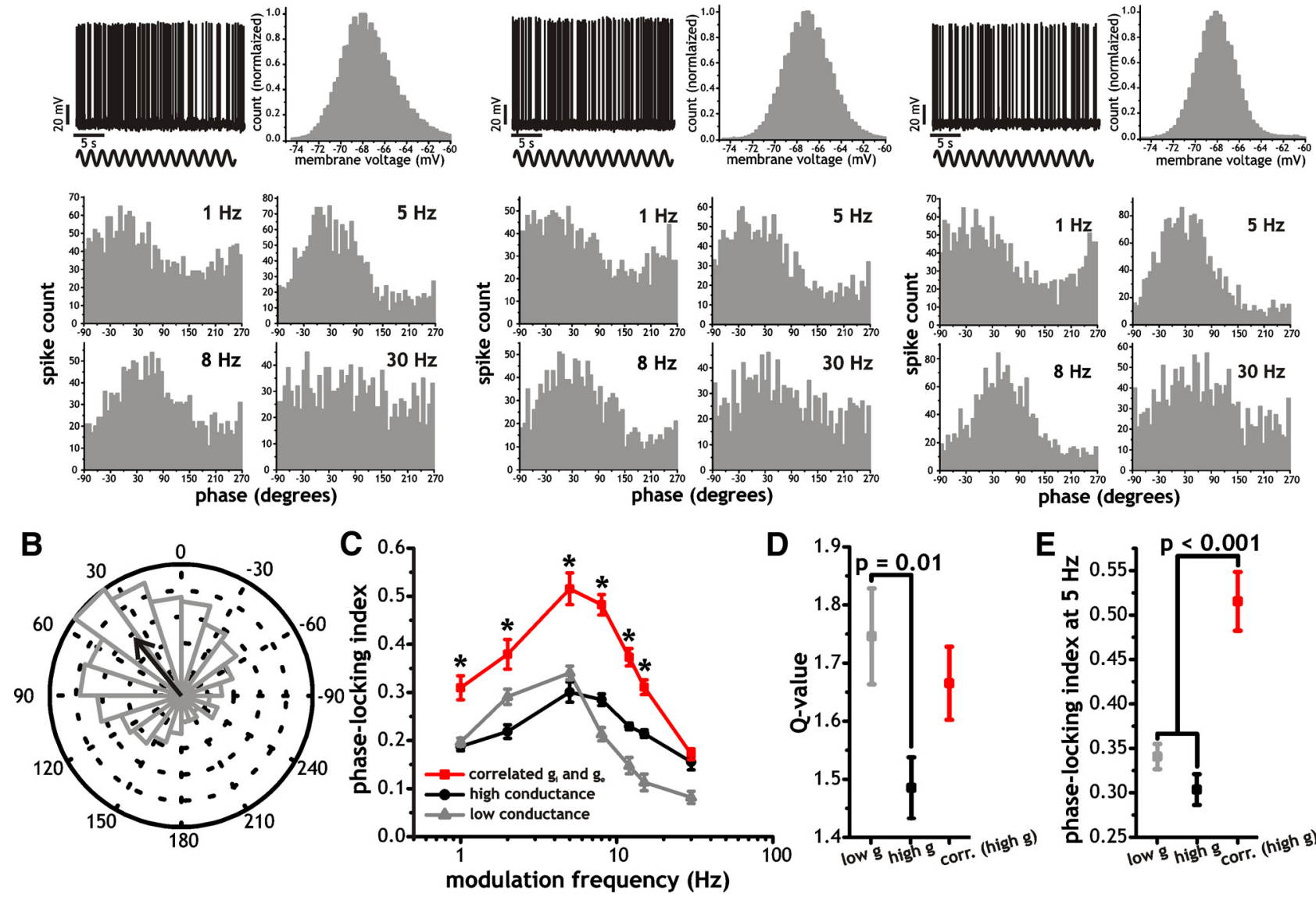

Figure 1. Changes in membrane conductance and correlations in inhibitory $\left(g_{i}\right)$ and excitatory $\left(g_{e}\right)$ synaptic conductance activity modulate spike phase-locking in MEC stellate cells. $\boldsymbol{A}$, Example spike response and subthreshold voltage histograms (top) as well as average spike phase histograms (bottom) for stellate cells given sinusoidal current input. For spike phase histograms, example shows a stellate cell driven by sinusoidal current inputs (frequency $=1,5,8,30 \mathrm{~Hz}$ ) in the presence background synaptic fluctuations generated with low $(\boldsymbol{A} \mathbf{1})$ and high $(\boldsymbol{A 2})$ membrane conductance or correlated (A3) artificial synaptic activity. B. Circular plot of binned spike phases (relative to a $5 \mathrm{~Hz}$ sinusoidal input) with low conductance background synaptic fluctuations. The arrow indicates the average phase of all spikes, while the length of the arrow indicates the strength of locking. $C$, Plot of the average spike phase-locking indices in response to sinusoidal current inputs of different frequencies $(1,5,8,12,15,30 \mathrm{~Hz})$ measured in the presence of the three different types of synaptic conductance-mediated membrane voltage fluctuations outlined in $\boldsymbol{A}$ (low, high, and correlated). Asterisks indicate significant difference between correlated versus low or high conductance conditions $(p<0.001)$. $\boldsymbol{D}$, Q-value (ratio of spike phase-locking indices at 5 and $1 \mathrm{~Hz}$ ) measured in the presence of background synaptic fluctuations generated with low (gray) and high (black) membrane conductance or correlated (red) artificial synaptic activity. $\boldsymbol{E}$, Phase-locking indices measured in response to $5 \mathrm{~Hz}$ sinusoidal input under low (gray) and high (black) membrane conductance or correlated (red) artificial synaptic activity.

threshold resonance in the theta band that is mediated by $I_{H}$ and generated via suppression to low $(1-3 \mathrm{~Hz})$ frequency inputs (Haas et al., 2007; Nolan et al., 2007; Heys et al., 2010). It is not known, however, if the established mechanisms supporting subthreshold resonance are related to those associated with spikefiring resonance observed in Figure 1. Past work on stellate cells from our group has also shown that increased membrane conductance significantly reduces subthreshold resonance by reducing the ability for $I_{H}$ to influence subthreshold voltage (Fernandez and White, 2008). We hypothesized that a reduction of subthreshold resonance either through a direct reduction of $I_{H}$ or a change in membrane conductance should have analogous effects on spike-firing resonance.

To establish whether $I_{H}$ plays a role in theta frequency selectivity of the spiking behavior through its effects on subthreshold membrane resonance, we used ZD7288 $(10 \mu \mathrm{M})$ to block $I_{H}$. The efficacy of ZD7288 was determined by measuring subthreshold membrane impedance before and after application of the drug (Fig. 2A1) in the subthreshold region of membrane voltage (mean voltage: $-74.9 \pm 0.5 \mathrm{mV}$, max: $-69.9 \pm 0.8 \mathrm{mV}$, min: $-78.8 \pm 0.5 \mathrm{mV}, n=11)$. We quantified the subthreshold reso- nance at theta using a ratio of the impedance values at 5 and $1 \mathrm{~Hz}$ (Q-value). This value was reduced from $1.62 \pm 0.1$ under control to $0.8 \pm 0.04$ under bath application of ZD7288 (Fig. 2A2; $p<$ 0.001 , one-way ANOVA, $n=11-24$ ). Application of the drug completely eliminated the subthreshold resonance response at theta frequencies by increasing the membrane impedance at low frequencies (Fig. 2A1). Increased membrane conductance added via dynamic clamp also reduced the Q-value. Unlike the ZD7288 condition, however, the cell retained a peak at theta albeit significantly smaller than under the low conductance condition (Fig. $2 A 2 ; 1.18 \pm 0.08$ vs $1.62 \pm 0.1, p=0.006$, one-way ANOVA, Tukey's test, $n=7-24$ ).

To measure spike phase-locking under ZD7288, we used the same frequency range and amplitude of sinusoidal current stimuli as in Figure 1. For these measures, we used a background consisting of either correlated or uncorrelated synaptic activity that included a mean conductance component. We observed that ZD7288 reduced the preference for spike phase-locking to theta inputs using either correlated or uncorrelated synaptic inputs. The loss of the spike resonance at theta was due to a significant increase in spike phase-locking strength to low-frequency (1-5 

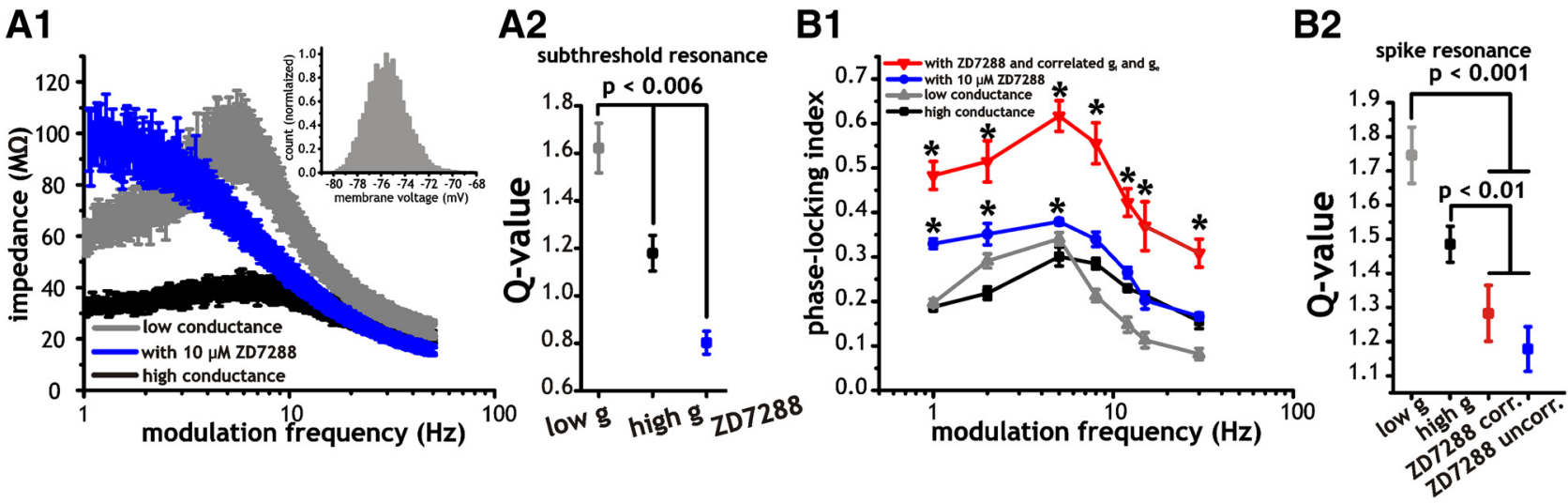

Figure 2. Frequency selectivity of stellate cells in the theta range is mediated by a hyperpolarization-activated cation current $\left(I_{H}\right)$ and is modestly affected by increased membrane conductance. A1, Plot of average membrane impedance measured under control (gray), with $10 \mu \mathrm{m} \mathrm{ZD7288} \mathrm{(blue)} \mathrm{or} \mathrm{with} \mathrm{increased} \mathrm{membrane} \mathrm{conductance} \mathrm{(black).} \mathrm{A2,} \mathrm{Plot} \mathrm{of} \mathrm{average} \mathrm{subthreshold} \mathrm{Q-values}$ (ratio of impedance or spike phase-locking indices at 5 and $1 \mathrm{~Hz}$ ) under each of the conditions outlined in $\mathbf{A 1}$. B1, Plot of the average spike phase-locking indices measured in response to sinusoidal current inputs of different frequencies $(1,5,8,12,15,30 \mathrm{~Hz}$ ) under low (gray) and high (black) conductance or in the presence of $10 \mu \mathrm{m}$ bath applied ZD7288 with (red) or without (blue) correlations in synaptic inputs. Asterisks indicate significant difference between spike phase-locking indices measured with ZD7288 (correlated or uncorrelated) versus control conditions ( $p<0.001)$. B2, Plot of average spike Q-values under each of the conditions outlined in B1.

$\mathrm{Hz}$ ) sinusoidal inputs under ZD7288 compared with low or high conductance conditions (Fig. 2B1; $p<0.01$, one-way ANOVA, Tukey's test, $n=5-12$ ). Consequently, with ZD7288, spike phase-locking to sinusoidal inputs between 1 and $8 \mathrm{~Hz}$ were not significantly different from each other (Fig. $2 B 1 ; p=0.32$, oneway ANOVA, $n=5-12$, uncorrelated inputs, $p=0.11$, one-way ANOVA, $n=6-9$, correlated inputs). Similar to the changes observed in subthreshold impedance, application of ZD7288 increased the spike phase-locking response to low-frequency input under both correlated (red line) and uncorrelated (blue line) synaptic stimuli compared with the control conditions (black line), which reduced the frequency preference at theta before the drop at higher frequencies (Fig. $2 B 1 ; p<0.001$, two-way ANOVA, Tukey's test, $n=5-9$ ). A comparison of the Q-values across low and high conductance as well as with ZD7288 using correlated or uncorrelated inputs indicated that changes in the subthreshold Q-value were qualitatively similar to those observed in the spike resonance response (Fig. 2, compare B2, A2). With ZD7288, Q-values associated with spike resonance were significantly reduced compared with measures taken under low or high conductance (Fig. $2 B 2 ; p<0.01$, one-way ANOVA, Tukey's test, $n=5-9$ ). Furthermore, as with control conditions, correlations in synaptic inputs in the presence of ZD7288 significantly increased spike phase-locking across all frequencies tested compared with ZD7288 and no correlations (Fig. 2B1: $p<0.001$, one-way ANOVA, Tukey's test, $n=$ 5-12). Hence, correlations enhance spike phase-locking but do not by themselves bestow spike resonance.

Overall, spiking resonance was consistently higher and more resistant to our manipulations using ZD7288 and increased membrane conductance than the subthreshold resonance. Nevertheless, these results indicate that $I_{H}$ and the mean membrane conductance play a significant role in shaping the spike-firing resonance via their influence on subthreshold resonance behavior. As a result, subthreshold resonance in stellate cells, which selectively amplifies sinusoidal inputs at theta by reducing membrane voltage response to low frequencies $(<4 \mathrm{~Hz})$, provides a larger voltage response that evokes more reliable spike phaselocking to theta inputs.
Correlations in $g_{i}$ and $g_{e}$ can selectively reduce the power of low-frequency input fluctuations

Although changes in membrane conductance and a reduction of $I_{H}$ had a significant impact on spike phase-locking behavior, the largest changes in locking strength were observed when $g_{i}$ and $g_{e}$ were correlated. Despite introducing a high conductance state, a background stimulus using correlations in synaptic inputs nearly doubled the spike phase-locking indices in response to inputs between 1 and $15 \mathrm{~Hz}$ and modestly increased theta selectivity relative to the high conductance stimulus using uncorrelated synaptic inputs (Fig. $1 C-E$ ). To better understand this result, we followed by looking into how the parameters in the OU process affected spike phase-locking.

A key parameter in our OU stimulus was the correlation strength ( $c$, from Eq. 3; see Materials and Methods). Intuitively, the correlation value controls how much $g_{i}$ and $g_{e}$ covary across time (Fig. 3A). To better understand how correlations influenced spike phase-locking, we evaluated the impact of different $c$ values (Fig. $3 A, B$ ). We hypothesized that reductions in correlation strength would gradually reduce the enhancement in spike phase-locking. For these experiments, we limited our measure of spike phase-locking to sinusoidal test input frequencies of $2 \mathrm{~Hz}$. We used correlation values between the random variables $\left(\chi_{i}\right.$ and $\chi_{e}$; see Materials and Methods) generating $g_{i}$ and $g_{e}$ activity of 1 , $0.8,0.4$, and 0 (Fig. $3 B$ ). Notice that these values generated effective peak correlations between $g_{i}$ and $g_{e}$ of $0.80,0.63,0.32$, and 0 due to differences in the time constants between $g_{i}$ and $g_{e}$ (Fig. $3 B$, inset). For all these experiments, the SD of membrane voltage was maintained at $\sim 1.5 \mathrm{mV}$. As shown in Figure $3 B$, decreasing the correlation strength between $g_{i}$ and $g_{e}$ led to progressively lower spike phase-locking indices (Fig. $3 B ; p<0.001$, one-way ANOVA, $n=8)$. A significant increase in spike phase-locking was maintained with $c=1$ and $c=0.8$ compared with the completely uncorrelated condition (Fig. $3 B ; p<0.05$, one-way ANOVA, Tukey's test, $n=8$ ). Thus, the strength of correlation between inhibition and excitation is important in modulating the spike phase-locking strength.

An additional parameter in our synaptic stimulus was the relative SDs of $g_{i}$ and $g_{e}$ fluctuations, denoted by $\rho\left(\rho=\sigma_{\mathrm{i}} / \sigma_{\mathrm{e}}\right)$. Recordings performed in vivo have shown that this ratio can vary 

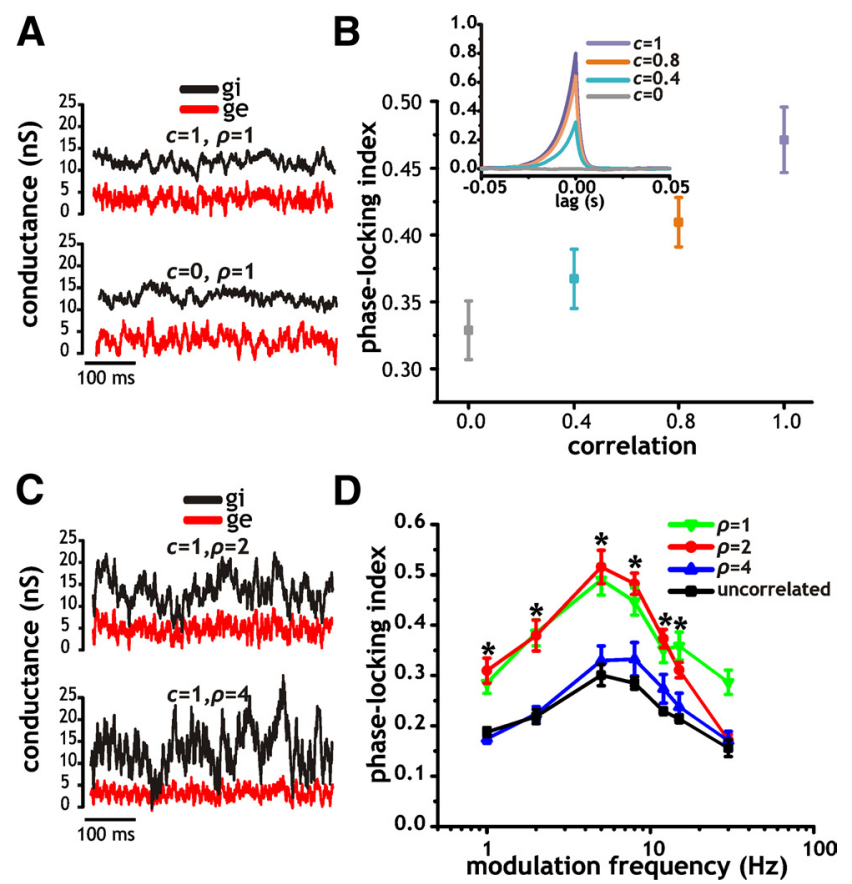

Figure 3. Correlations strength $(c)$ and the relative SD $(\rho)$ between inhibitory $\left(g_{i}\right)$ and excitatory $\left(g_{e}\right)$ synaptic conductance fluctuations modulate spike phase-locking in stellate cells. $A$, Example traces of inhibitory (black) and excitatory (red) synaptic conductances associated with artificial synaptic stimuli using $c=1$ (top) and $c=0$ (bottom) with a fixed $\rho$ value of 1. $\boldsymbol{B}$, Plot of the average spike phase-locking indices measured in response to sinusoidal current inputs at $2 \mathrm{~Hz}$ using different correlation strengths $(0,0.4,0.8$ and 1$)$. Inset shows crosscorrelation function between $g_{i}$ and $g_{e}$ under each of the correlation strength values used. $C$, Example traces of inhibitory (black) and excitatory (red) synaptic conductances associated with artificial synaptic stimuli using $\rho=2$ (top) and 4 (bottom) with $c=1$. D, Plot of the average spike phase-locking indices in response to sinusoidal current inputs of different frequencies (1, $5,8,12,15,30 \mathrm{~Hz}$ ) measured in the presence of four different types of synaptic conductancemediated membrane voltage fluctuations $(c=1 ; \rho=1,2,4$ and $c=0)$. Asterisks indicate significant difference between correlated with $\rho=1$ or 2 versus uncorrelated and $\rho=4$ ( $p<$ 0.001).

as a function of network state (Rudolph et al., 2007; Wu et al., 2008). Hence, depending on the value of $\rho$, the moment-bymoment synaptic current fluctuations in neurons can deviate from a perfect balance and hence change how correlations affect the moment-by-moment balancing of synaptic inhibition and excitation. We tested the effects of using three different values of $\rho(\rho=1,2$, and 4$)$, while maintaining the correlation between $g_{i}$ and $g_{e}$ fixed at 1 (Fig. $3 C, D$ ). To our surprise, the choice of $\rho$ had a strong effect on spike phase-locking behavior even though the $\mathrm{SD}$ of the membrane voltage fluctuations was identical to our previous conditions. Notably, smaller inhibitory current fluctuations relative to excitation when $\rho$ was set to 1 or 2 were associated with enhanced spike phase-locking across different sinusoidal input frequencies $(1-15 \mathrm{~Hz})$ compared to when $\rho$ was set to 4 or when $g_{i}$ and $g_{e}$ were uncorrelated (Fig. $3 D ; p<0.001$, one-way ANOVA, Tukey's test, $n=20$ ).

In summary, the ability for correlations in synaptic activity to influence the spike phase-locking response was a result of both the correlation strength and the relative magnitudes of $g_{i}$ and $g_{e}$ fluctuations. Although our experimental results pointed to the importance of both these two parameters, we did not have a mechanistic understanding of how these factors affected spike phase-locking and shaped membrane voltage and synaptic inputs current fluctuations. We proceeded to analyze a simple model of membrane voltage with synaptic conductance inputs inducing fluctuations similar to our experiments.

\section{Correlations and the SD of $g_{i}$ and $g_{e}$ shape the power spectrum of synaptic current fluctuations}

As a first step to addressing how synaptic conductance input parameters ( $c, \rho$, and synaptic kinetics) determine neuronal output, we studied a simplified model of membrane voltage in the form of an RC circuit. We wanted to precisely relate synaptic conductance fluctuations with changes in the statistics of membrane voltage fluctuations. A change in voltage could then be used to understand how correlations in synaptic conductances influence spike output. To start, we focused on the relationship between conductance inputs and membrane current and how $c$ and $\rho$ change total net current delivered via synaptic conductance inputs. Through an understanding of how conductance inputs and correlations influence net synaptic current we could better understand how they change membrane voltage fluctuations and ultimately spike discharge.

We modeled synaptic conductance inputs as two OU processes identical to those used in our experiments and set the time constant of the RC circuit to a value within the range measured for stellate cells (membrane time constant $=10 \mathrm{~ms}: C=1 \mu \mathrm{F} /$ $\mathrm{cm}^{2}$ and $\left.g_{\text {leak }}=0.1 \mathrm{mS} / \mathrm{cm}^{2}\right)$ and used mean $g_{i}\left(\bar{g}_{i}\right)$ and $g_{e}\left(\bar{g}_{e}\right)$ values of 0.2 and $0.05 \mathrm{mS} / \mathrm{cm}^{2}$, respectively. The SD of $g_{e}$ was set to $0.01 \mathrm{mS} / \mathrm{cm}^{2}$, while the SD of $g_{i}$ was a multiple of 1,2 , or 4 of this value (i.e., $\rho=1,2$, or 4 ).

Previous theoretical work has shown that correlations between $g_{i}$ and $g_{e}$ can reduce the size of net synaptic current fluctuations (sum of inhibitory and excitatory currents) (Renart et al., 2010). To quantify the size or power in synaptic current input fluctuations across different frequencies, we calculated the power spectrum of the total current delivered to the RC circuit through the OU processes. Any potential reduction in the size of current fluctuations would be indicated as a reduction in power within a particular frequency range. We used an analytical approximation of our model and calculated the spectrum for total synaptic current using different parameters for the OU processes (see Materials and Methods).

For our analytical results, we initially focused on synaptic inputs with parameters set to $\rho=2$ or 4 while keeping $c=1$. We found that the shape of the power spectrum for total synaptic input current depends strongly on the value of $\rho$ (Fig. $4 A$ ). When $g_{i}$ and $g_{e}$ are correlated and $\rho$ is 2 , the resulting power spectrum for total synaptic current is strongly non-monotonic with a large reduction in power at low frequencies (Fig. 4A1). On the other hand, when $\rho$ is 4 , the power spectrum has increased power at low frequencies and is monotonic in shape (Fig. 4A2). Hence, with correlations, synaptic conductance inputs with low-pass spectra (i.e., OU processes with physiological time constants) can produce net current output fluctuations with a bandpass spectrum (Fig. 4A1).

To understand how differences in the amount of power at low frequencies in the total synaptic current arise, we plotted the spectra for the individual inhibitory and excitatory currents (Fig. 4B). With $\rho=2$, the amount of power at low frequencies $(0-20 \mathrm{~Hz})$ in inhibitory and excitatory currents is well matched (Fig. $4 B 1$, red and black line in gray shaded area). At frequencies $>20 \mathrm{~Hz}$, however, the amount of power in excitatory current fluctuations is greater than that for inhibitory fluctuations. In contrast, a $\rho$ value of 4 results in much greater power in inhibitory current fluctuations at low frequencies. Thus, as indicated by the power spectra, inhibitory and 

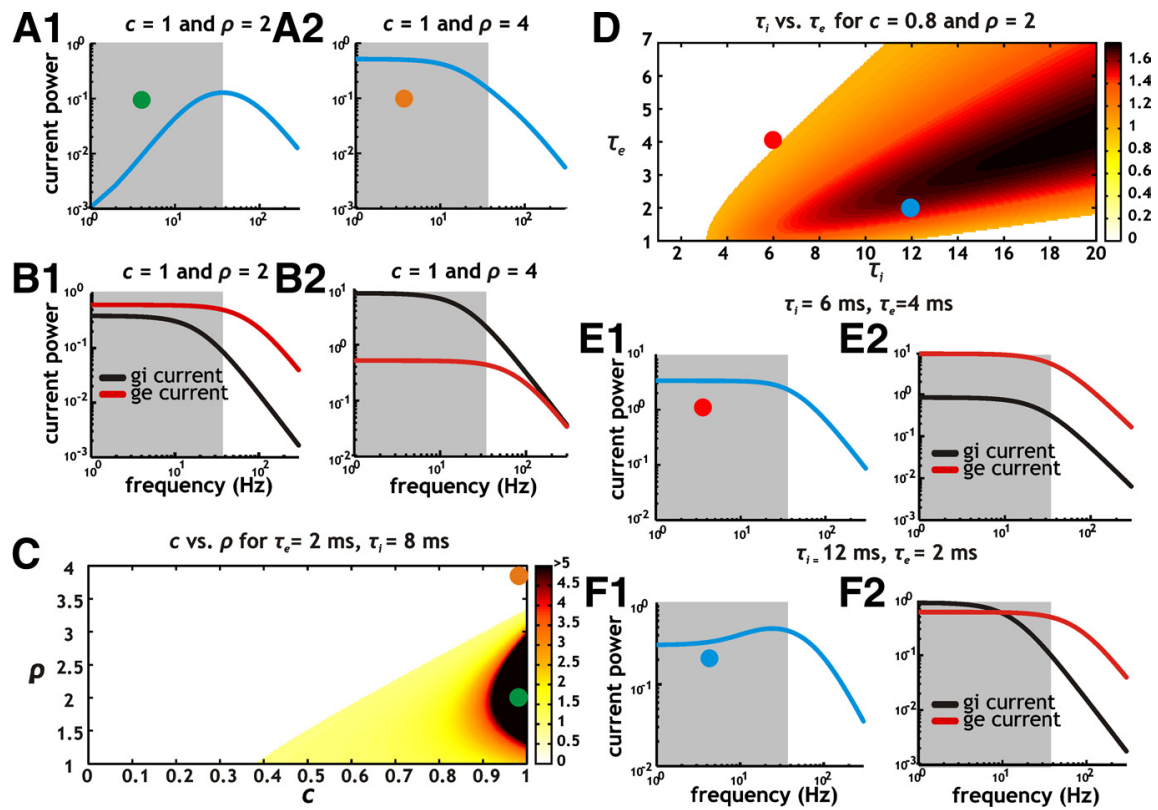

Figure 4. Reduction of low-frequency input fluctuations depends on correlation strength $(c)$, SD ratio $(\rho)$ and synaptic decay time constants of $g_{i}$ and $g_{e}$ conductance fluctuations. A, Analytically derived power spectra for total synaptic current using $c=1$ with $\rho=2(\boldsymbol{A 1})$ and $\rho=4(\boldsymbol{A 2})$. Parameter locations in color map $(\boldsymbol{C})$ from which power spectra were calculated are indicated with a green $(\rho=2)$ and orange $(\rho=4)$ dot. $\boldsymbol{B}$, Power spectra for inhibitory (black) and excitatory (red) synaptic currents associated with $\boldsymbol{A}$ using $c=1$ along with $\rho=2(B 1)$ and $\rho=4(B 2)$. C, Color map indicating degree of non-monotonicity (increased non-monotonicity indicated with dark red) as a function of c and $\rho$ using time constants for inhibition $\left(\tau_{i}\right)$ and excitation $\left(\tau_{e}\right)$ of 8 and $2 \mathrm{~ms}$, respectively. The non-monotonicity index was calculated by taking the ratio of power at the peak frequency and power at $0 \mathrm{~Hz}$. In the case of monotonic spectra, the index was set to 0 . Non-monotonicity indices $>5$ were capped at a value of 5 . $D$, Color map indicating degree of non-monotonicity as a function of $\tau_{i}$ and $\tau_{e}$ using $c=0.8$ and $\rho=2$. Non-monotonicity was calculated using an index identical to $\boldsymbol{C}$. $\boldsymbol{E}$, Power spectra of total synaptic current input (E1) as well as the individual inhibitory (E2, black) and excitatory (E2, red) currents resulting from using $\tau_{i}$ and $\tau_{e}$ of 6 and $4 \mathrm{~ms}$, respectively. The power spectrum profile for total synaptic current input (E1) is monotonic ( $\boldsymbol{E}$ 1, gray shaded region). $\boldsymbol{F}$, Power spectra of total synaptic current input (F1) as well as the individual inhibitory ( $\boldsymbol{F} 2$, black) and excitatory ( $\boldsymbol{F} 2$, red) currents resulting from using $\tau_{i}$ and $\tau_{e}$ of 12 and $2 \mathrm{~ms}$, respectively. Parameter locations in color map (D) from which power spectra were calculated are indicated with a red ( $\tau_{i}$ and $\tau_{e}$ of 6 and $4 \mathrm{~ms}$ ) and blue ( $\tau_{i}$ and $\tau_{e}$ of 12 and $2 \mathrm{~ms}$ ) dot. Notice that the power spectrum profile for total synaptic current input (F1) is nonmonotonic and has a selective reduction in power at low frequencies ( $\boldsymbol{F 1}$, gray shaded region) compared with the case shown in $\boldsymbol{E}$.

excitatory current fluctuations are closely matched in terms of magnitude at low frequencies with $\rho=2$ but not with $\rho=4$.

Fundamentally, the difference in power at low frequencies is related to the scaling of inhibitory and excitatory fluctuations with different $\rho$ values. A lower $\rho$ value is associated with smaller inhibitory fluctuations. Conversely, when $\rho=4$, inhibitory fluctuations are larger and have much more power than excitatory fluctuations at low frequencies. Hence, the combination of a similar magnitude at low frequencies with $\rho=2$ and high temporal correlation in $g_{i}$ and $g_{e}$ leads to mutual cancellation at low frequencies. As a consequence, inhibitory and excitatory current fluctuations cancel at low but not high frequencies. For this reason, the total synaptic current power spectrum has reduced power at low frequencies when $\rho=2$ (Fig. 4A1).

To quantify the dependence of the synaptic current power spectrum on the parameters $c$ and $\rho$, we generated a color map showing the degree of non-monotonicity (a measure of the selective loss of low-frequency power) as a function of $c$ and $\rho$. Whenever we found a non-monotonic power spectrum, we used the ratio of peak power at a nonzero frequency (usually near $30 \mathrm{~Hz}$ ) with the value of power at $0 \mathrm{~Hz}$. Values $>1$ indicate a nonmonotonic power spectrum and are indicated with darker shades of orange and red in Figure 4C. Increasing the correlation strength between $g_{i}$ and $g_{e}$ with $\rho$ values near 2 results in increasingly non-monotonic synaptic current power spectra
(Fig. 4C). Conversely, for low correlation strengths $(<0.5)$ and large $\rho$ values $(>3)$, current power spectra are largely monotonic (Fig. 4C).

The distribution of power in inhibitory and excitatory current fluctuations is also influenced by the kinetics of synaptic decay. Faster synaptic processes have a larger fraction of power at higher frequencies, while slow processes have more power at low frequencies. Time constants for inhibition and excitation occur over a relatively narrow range of values in neurons, with inhibition being normally slower than excitation. Because of this we were interested in how different synaptic decay kinetics influence cancellation at low frequencies. Thus, in addition to $\rho$, the time constants of synaptic inhibition and excitation $\left(\tau_{i}\right.$ and $\tau_{e}$ ) should influence the degree of non-monotonicity in the synaptic current power spectrum in the presence of correlations.

To start, we froze the correlation strength and $\rho$ value at 0.8 and 2 , respectively, and varied $\tau_{i}$ and $\tau_{e}$. As indicated, non-monotonic synaptic power spectra are more likely when $\tau_{i}$ is larger than $\tau_{e}$ (Fig. $4 D$ ). For cases when $\tau_{i}$ and $\tau_{e}$ are similar (e.g., 6 and 4 ms; Fig. 4E1), the power spectrum is monotonic because both inhibitory and excitatory conductances have a similar distribution of power across different frequencies (Fig. 4E2). As a result, when coupled with a $\rho$ value of 2 , inhibition has uniformly less power at all frequencies relative to excitation such that there is no selective cancellation of inhibitory and excitatory current fluctuations with respect to frequency. On the other hand, when $\tau_{i}$ is larger than $\tau_{e}$ (e.g., 12 and $2 \mathrm{~ms}$; Fig. $4 F)$, the distribution of power is different for inhibition and excitation (Fig. 4F2). Relative to inhibition, excitation has much more power at higher frequencies but similar power at low frequencies (Fig. 4F2). For this reason, inhibitory and excitatory synaptic current fluctuations selectively cancel at low frequencies and a non-monotonic profile for the power spectrum is generated (Fig. 4F1). Hence, for a wide range of physiologically relevant parameters the power spectrum of current input fluctuations has substantially reduced power at low frequencies when $g_{i}$ and $g_{e}$ are correlated.

\section{Experimental verification of theoretical results}

Our analytical results indicated that correlations in $g_{i}$ and $g_{e}$ shape the spectra of background current input fluctuations and could thus play a role in shaping membrane voltage and the spike phase-locking response. Before testing how the shape of the power spectrum affected the spike phase-locking response, however, we needed to verify that our data also generated reduced power in low-frequency fluctuations by measuring the power spectra of inhibitory and excitatory currents delivered via dynamic clamp in stellate cells.

To avoid contamination from spikes in the measure of power spectra, we held membrane voltage slightly below spike threshold 

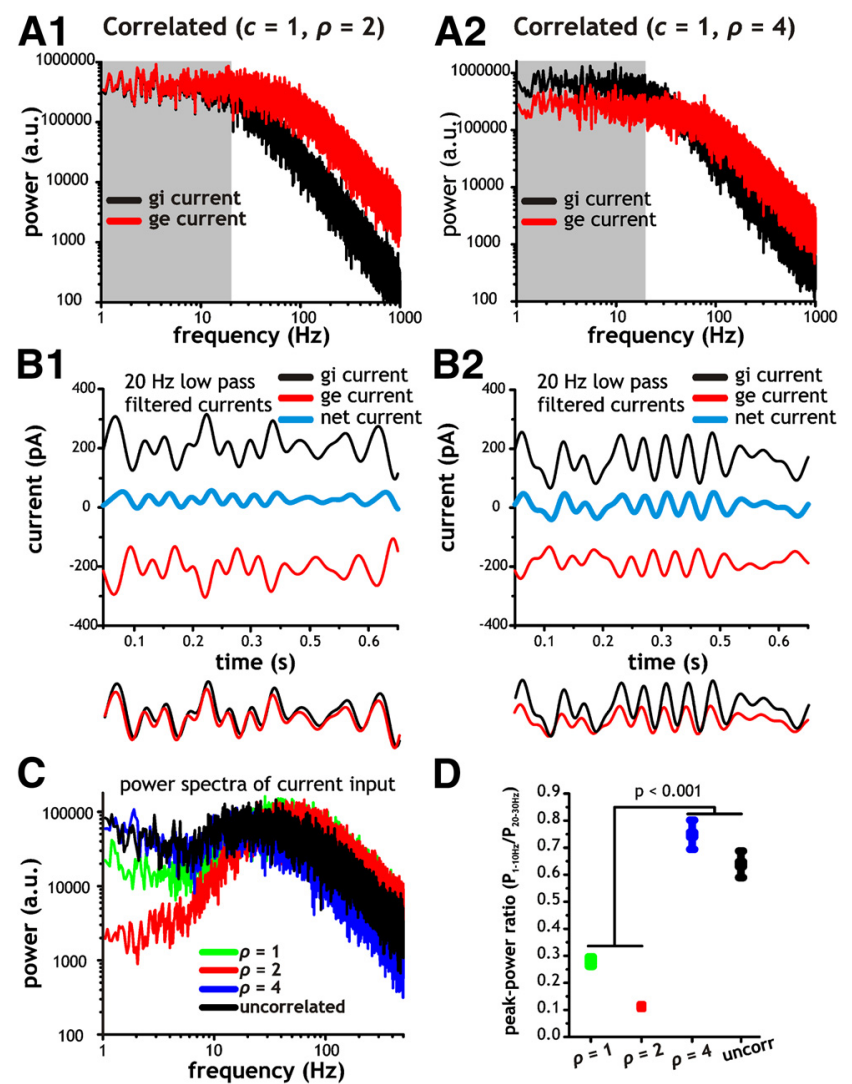

B2

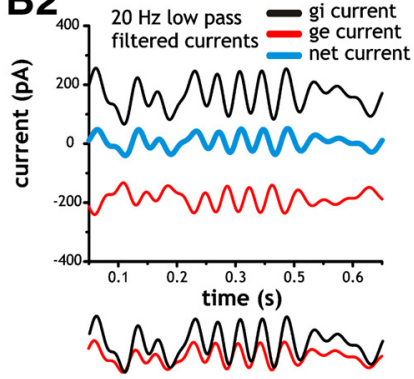

D

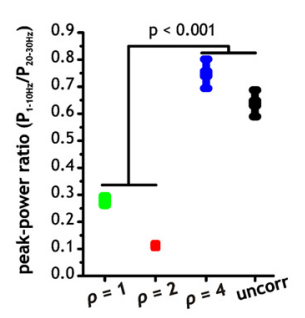

Figure 5. Correlations in synaptic conductance inputs induce cancellation of inhibitory and excitatory currents at low frequencies. $\boldsymbol{A}$, Example power spectra of inhibitory (black) and excitatory (red) currents associated with artificial synaptic stimuli using $\rho=2(\boldsymbol{A} 1)$ and 4 (A2). $\boldsymbol{B}$, Low-pass filtered (20 Hz cutoff) traces of inhibitory (black) and excitatory (red) currents with $\rho=2$ (B1) and 4 (B2). The blue traces indicate the net instantaneous difference between inhibitory and excitatory currents within the $20 \mathrm{~Hz}$ cutoff range. Below the plots, the filtered traces for the corresponding inhibitory and excitatory have been superimposed (excitatory current is mirrored) for the purposes of comparison. C, Example power spectra of total synaptic current delivered via dynamic clamp. D, Plot of average ratio of total power between 1 and $10 \mathrm{~Hz}$ versus 20 and $30 \mathrm{~Hz}\left(\mathrm{P}_{1-10 \mathrm{~Hz}} / \mathrm{P}_{20-30 \mathrm{~Hz}}\right)$ under each of the four different background synaptic conditions. Notice that as in our analytical results (Fig. 4), conditions with $\rho=1$ and 2 generate total synaptic input current fluctuations with a substantial reduction in low-frequency power.

and calculated power spectra based on $50 \mathrm{~s}$ long synaptic current input traces. For these experiments we did not include the sinusoidal input current delivered during measures of spike-phase locking. For contrast, we compared the power spectra and time series of inhibitory and excitatory currents in a condition predicted by our analysis to have high $(\rho=4)$ and low $(\rho=2)$ amounts of power in low-frequency synaptic current fluctuations (Fig. $4 A$ ). With $\rho=2$, the power of inhibitory and excitatory currents at frequencies at or below $20 \mathrm{~Hz}$ was matched nearly perfectly (Fig. 5A1, gray shaded region). At frequencies $>20 \mathrm{~Hz}$, the amount of power in excitatory current was greater than that for inhibitory current due to excitation being faster than inhibition ( $2 \mathrm{~ms}$ vs $8 \mathrm{~ms}$ ). In contrast, a $\rho$ value of 4 resulted in greater power in the inhibitory current at low frequencies $(<20 \mathrm{~Hz})$ and greater power in the excitatory current at high frequencies $(>20$ $\mathrm{Hz}$ ). Under this condition, inhibitory and excitatory currents were not matched in power across any considerable frequency range (Fig. 5A2). Hence, consistent with our mathematical results, the power spectra of inhibitory and excitatory inputs show differential amounts of power across frequency depending on the value of $\rho$.
We proceeded to filter the current traces using a $20 \mathrm{~Hz}$ lowpass filter to highlight the frequency range in which the power of inhibitory and excitatory currents differed depending on our choice of $\rho$. Within this frequency range, the magnitudes of inhibitory and excitatory currents associated with $\rho=2$ were nearly the same size (Fig. 5B1) and hence better balanced than $\rho=4$ in the same frequency range (Fig. 5B2). For this reason, and as predicated by our analytical results, the reduction in current input fluctuations at low frequencies with $\rho=2$ (Fig. $5 B 1$, blue trace) is a result of inhibitory and excitatory current fluctuations cancelling within this frequency range when $g_{i}$ and $g_{e}$ are correlated.

Next, we performed power spectrum analyses of the total artificial synaptic input current delivered via dynamic clamp. As predicated by our analytical results, correlations in $g_{i}$ and $g_{e}$ with $\rho$ values of 1 and 2 produced highly non-monotonic power spectra with significantly less power between 1 and $10 \mathrm{~Hz}$ versus 20 and $30 \mathrm{~Hz}\left(\mathrm{P}_{1-10 \mathrm{~Hz}} / \mathrm{P}_{20-30 \mathrm{~Hz}}\right.$; Fig. $5 C, D ; p<0.001$, one-way ANOVA, Tukey's test, $n=9)$. The cancellation at low frequencies with $\rho=1$ or 2 occurs because under these conditions inhibitory and excitatory synaptic currents (due to $\rho, c$, and synaptic kinetics) are specifically balanced and matched at low frequencies.

\section{Reducing power of low-frequency $(0-20 \mathrm{~Hz})$ membrane voltage fluctuations increases spike phase-locking to theta inputs}

Next, we sought to understand how the reduction in lowfrequency current input fluctuations $(0-20 \mathrm{~Hz})$ led to an increase in spike phase-locking at theta frequencies. Past theoretical work has shown that the frequency content of background fluctuations can influence spike-phase locking. Specifically, a reduction of power in background fluctuations at high frequencies can enhance locking in that same range (Brunel et al., 2001; Wei and Wolf, 2011). In our case, we observed that the low-frequency range in which synaptic input fluctuations were reduced overlapped with the sinusoidal input frequencies in which correlations substantially improved spike phase-locking $(1-15 \mathrm{~Hz})$ to sinusoidal inputs. In order for a reduction in synaptic current input fluctuations at low frequencies to affect spike phase-locking behavior, however, it must also change the size of voltage fluctuations that drive probabilistic spike discharge. We reasoned that a reduction in random low-frequency membrane voltage fluctuations would reduce interference between background voltage fluctuations and the sinusoidal input signal.

To establish if membrane voltage fluctuations were indeed being selectively reduced at low frequencies with correlations and $\rho$ values of 1 and 2 , we held membrane voltage slightly below spike threshold and calculated power spectra based on $50 \mathrm{~s}$ long subthreshold voltage traces. As with synaptic current input, we found that the power spectrum of membrane voltage depended on both the presence of correlations and the value of $\rho$ between $g_{i}$ and $g_{e}$ (Fig. 6). When $g_{i}$ and $g_{e}$ were correlated and $\rho$ was equal to 1 or 2 , the resulting power spectra of voltage were strongly nonmonotonic with a large reduction in power at low frequencies (Fig. 6). On the other hand, when $\rho$ was 4 or $g_{i}$ and $g_{e}$ were uncorrelated, the power spectra had increased power at low frequencies and were monotonic in shape (Fig. 6). Thus, correlated $g_{i}$ and $g_{e}$ activity with $\rho$ of 1 or 2 reduced the power of membrane voltage fluctuations significantly between 1 and $10 \mathrm{~Hz}$, relative to the other two conditions ( $p<0.03$, one-way ANOVA, Tukey's test, $n=9)$.

If all that is required to increase spike phase-locking at low frequencies is a reduction in voltage fluctuations resulting from reduced power in input current, and not other complexities as- 

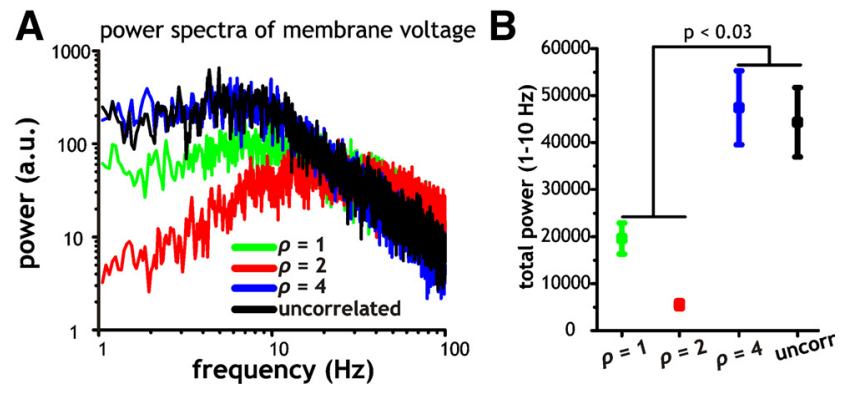

Figure 6. Correlations in synaptic conductance inputs selectively reduce the power of membrane voltage fluctuations at low frequencies. $\boldsymbol{A}$, Example power spectra of membrane voltage fluctuations mediated by artificial synaptic activity incorporating correlated $(c=1)$ or uncorrelated $g_{i}$ and $g_{e}$ fluctuations implemented with $\rho=1,2$, and 4 . $\boldsymbol{B}$, Plot of average integrated power between 1 and $10 \mathrm{~Hz}$ under each of the four different synaptic stimuli used in $\boldsymbol{A}$. Notice that membrane voltage fluctuations generated with $\rho=1$ or 2 contained significantly less power at low frequencies compared with stimuli generated with uncorrelated $g_{i}$ and $g_{e}$ or $\rho=4$.

sociated with conductance-based inputs, it should be possible to increase spike phase-locking by constructing background fluctuations with a specific spectra incorporating reduced power at low frequencies. Hence, to test our hypothesis, we constructed an artificial current signal having a non-monotonic current input power spectrum similar to that generated when $g_{i}$ and $g_{e}$ were correlated with $\rho=1$ or 2 (Fig. $7 A$, gray line). The current signal was constructed by applying a bandpass filter to a white noise current signal (see Materials and Methods). As before, we embedded a separate sinusoidal current input (20 pA peak-to-peak amplitude) with the background current noise to test spike phase-locking. To account for the increase in mean conductance associated with our previous OU processes, we added a separate linear leak conductance equal in magnitude to the total mean conductance added previously (15 nS). We then compared spike phase-locking to the sinusoidal input with those acquired using background noise with a monotonic current power spectrum (Fig. 7A, black line).

Using the same procedure as before, we found that spike phase-locking was significantly modulated by our choice of background noise (bandpass vs low-pass) and sinusoidal input frequency (Fig. $7 B, C ; p<0.001$, two-way ANOVA, $n=5$ ). As predicted, background membrane voltage fluctuations generated through bandpass current input fluctuations were associated with significantly higher phase locking indices between 1 and 15 $\mathrm{Hz}$ (Fig. $7 B ; p<0.002$, one-way ANOVA, Tukey's test, $n=5$ ). Thus, the increase in spike phase-locking to sinusoidal signals between 1 and $15 \mathrm{~Hz}$ is a result of a reduction in power of random current and voltage fluctuations that increase the signal-to-noise ratio in that frequency range. Thus, our results show that stellate cells show a strong preference for spike phase-locking to theta rhythms and that this locking can be modulated by statistical qualities of inputs that are known to vary in vivo.

\section{Discussion}

In summary, we find that MEC stellate cells have a strong and robust preference for spike phase-locking to theta inputs under both increased membrane conductance and probabilistic spike discharge. As with subthreshold resonance, the preference for theta inputs in spike output is established largely by $I_{H}$. The magnitude of locking to theta inputs is also controlled by the statistical properties of background synaptic activity. By altering the spectral properties of voltage fluctuations, we show that synaptic correlations have a substantial impact on spike output response to time-varying stimuli. Balanced and correlated excitatory and inhibitory inputs with realistic decay kinetics cancel in the $0-20$ $\mathrm{Hz}$ frequency range. For this reason, small probe inputs in this frequency band can entrain stellate cells extremely effectively.

\section{Theta frequency preference in stellate cells}

Under all conditions tested, we found that stellate cell spike activity was better modulated by theta range sinusoidal current inputs. This result is consistent with previous modeling work indicating that very strong subthreshold resonance behavior carries into the spike output under a high noise or a fluctuationdriven regime (Richardson et al., 2003).

Past work from our group established that increased membrane conductance associated with synaptic input completely eliminates autonomous spike train oscillations and reduces subthreshold resonance at theta frequencies in stellate cells (Fernandez and White, 2008). The discrepancy with our previous results in stellate cell arises for two principal reasons. First, the ability to generate autonomous oscillatory activity is qualitatively different from the ability to amplify or respond preferentially to certain input frequencies. Thus, while stellate cells do not generate oscillations in response to unmodulated stochastic inputs under high membrane conductance, they do respond preferentially to input modulated at theta under these same conditions. Second, we observed that spike resonance was less sensitive to changes in membrane conductance than the subthreshold resonance behavior. This may arise from the fact that increased membrane conductance did not completely eliminate subthreshold resonance. Further, increased synaptic conductance has a smaller effect near threshold because of substantial $\mathrm{Na}^{+}$current activation, which acts to amplify resonance and intrinsic properties immediately below spike threshold (Alonso and Llinás, 1989; Burton et al., 2008). Finally, spike-dependent mechanisms may also contribute to the expression of spike resonance as shown in CA1 pyramidal cells (Broicher et al., 2012) and suggested in past work from stellate cells (Nolan et al., 2007; Fernandez and White, 2008).

\section{Comparison with in vivo stellate cell spike activity}

Stellate cell spike discharge in vivo occurs at relatively low and variable rates and is dominated by strong spike phase-locking to the local theta field potential (Alonso and García-Austt, 1987; Frank et al., 2001; Quilichini et al., 2010; Burgalossi et al., 2011). Importantly, mean spike rate data indicates a marked absence of repetitive spike discharge at theta or any form of pacemaking activity (Frank et al., 2001; Quilichini et al., 2010; SchmidtHieber and Häusser, 2013). Whether the spike temporal autocorrelations indicate significant oscillations at theta seems more variable. Some studies indicate an absence of rhythmicity in the autocorrelation (Frank et al., 2001; Quilichini et al., 2010), while others indicate rhythmic activity at theta (Brandon et al., 2011; Koenig et al., 2011). All these studies, however, indicate low average spike discharge rates $(<3 \mathrm{~Hz})$ that are significantly below theta. Hence, stellate cell spike activity locks strongly to theta inputs despite not firing at theta frequencies. This apparent discrepancy arises because the ability to lock spike discharge to a particular input frequency under stochastic membrane fluctuations is distinct from the continuous generation of spikes at a given spike rate and the ability to generate oscillations. In fact, recent intracellular in vivo recordings from stellate cells indicate an absence of intrinsically generated subthreshold membrane 
voltage oscillations but the ability to lock to the theta field potential (SchmidtHieber and Häusser, 2013).

Spike phase-locking requires only that the phase between the sinusoidal input and spike time be consistent across different cycles of the input. Although this result suggests that stellate cells are unlikely to provide pacemaking activity to downstream cells, it does suggest a potential role in amplifying or maintaining spike phase-locking to network theta rhythms originating from other cells. Furthermore, it indicates that a contribution to network theta by stellate cell can be achieved through very strong spike locking at theta resulting from $I_{H}$ and a reduction in low-frequency membrane voltage fluctuations by correlations in synaptic inputs.

The properties of $I_{H}$ are also known to vary systematically along the dorsalventral axis of the MEC (Giocomo et al., 2007; Garden et al., 2008). We would expect this gradient to affect the peak and/or the frequency preference of the spike phase-locking relationship. Although in HCN1 knock-out mice grid cells maintain spike phase-locking to the theta field potential (Giocomo et al., 2011), it is difficult to interpret these results in the context of a frequency selectivity index as locking to different frequencies in the local field potential have not been tested systematically in vivo. The gradient has also been shown to affect integration of synaptic inputs (Garden et al., 2008). Thus, it is possible that differences in spike timing along dorsal-ventral axis are reflected in different spike phase-locking responses due to differences in the membrane time constant, $I_{H}$ kinetics, and overall response to synaptic inputs.

\section{Functional consequences of correlations in $g_{i}$ and $g_{e}$}

Network models incorporating feedback inhibition can reliably generate robust correlations between excitation and inhibition, which reduce the size of voltage fluctuations through mutual cancellation and decrease spike discharge rate (Vogels and Abbott, 2009; Renart et al., 2010; Sceniak and Sabo, 2010; Economo and White, 2012). However, in vivo recordings indicate the presence of large voltage fluctuations that seem undampened by the correlations in synaptic inhibition and excitation (Destexhe and Paré, 1999; Crochet and Petersen, 2006). Furthermore, different network states are associated with different forms of subthreshold membrane voltage fluctuations. For example, states of wakefulness are associated with a selective decrease in power of low-frequency membrane voltage fluctuations (Crochet and Petersen, 2006; Poulet and Petersen, 2008; Okun et al., 2010; Constantinople and Bruno, 2011; Crochet et al., 2011). Our results suggest that these changes in spectra can arise through correlations between $g_{i}$ and $g_{e}$ using a physiological range of synaptic time constants and changes in the relative SD values.

A proposed function for synaptic correlations is that they reduce the size of membrane voltage fluctuations and/or shorten the duration of excitatory events (Pouille and Scanziani, 2001;
B

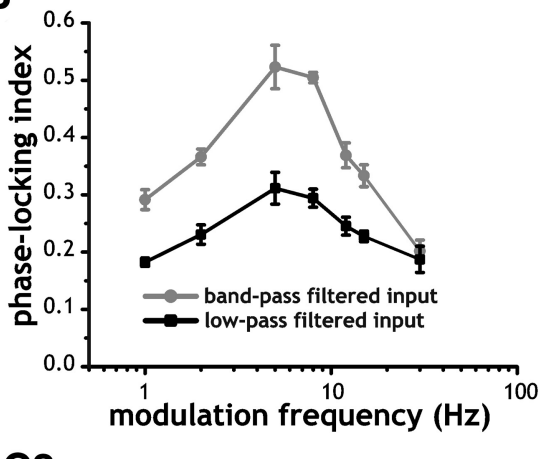

C2

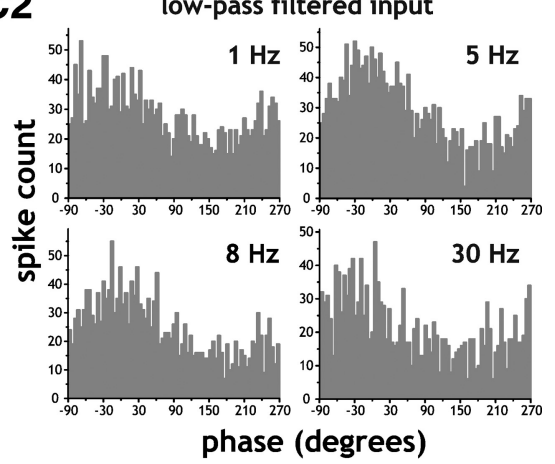

phase (degrees)

Wehr and Zador, 2003; Liu et al., 2010; Renart et al., 2010; Sceniak and Sabo, 2010), thereby increasing the temporal precision of spike output. Our results add to this and emphasize the role of correlations in modulating the spike phase-locking response. As with levels of mean conductance and the size of noisy fluctuations in our previous work (Fernandez and White, 2008, 2010; Fernandez et al., 2011), we now indicate that the correlation properties of synaptic inputs can have a large impact on cellular input-output behavior.

Computational work has also shown that a reduction of membrane voltage fluctuations resulting from correlations can be used to implement a decorrelation of spike activity in neighboring cells (Renart et al., 2010). Mutual cancellation of excitation and inhibition can be used to reduce membrane voltage and spike train correlations between neighboring cells arising from spatially diffuse negative feedback. Whereas our work indicates that correlations in inhibition and excitation can reduce the size of membrane voltage fluctuations, the magnitude and frequency range of the reduction is highly dependent on the SD and time constants of $g_{i}$ and $g_{e}$ fluctuations.

Although under our experimental conditions we used only a single pair of time constants for inhibition and excitation (8 and $2 \mathrm{~ms}$ ), our theoretical results indicate that the specific reduction of power in low-frequency voltage fluctuations occurs under a variety of parameters where inhibition is slower than excitation. The specific reduction of low-frequency voltage fluctuations is likely to be important for neurons as responses to higher frequencies are limited by the membrane time constant, synaptic time constants and the spike generation mechanism (Brunel et al., 
2001; Fourcaud-Trocmé et al., 2003; Wei and Wolf, 2011; Broicher et al., 2012).

Recent spectral analyses of membrane voltage in vivo have also revealed that membrane voltage dynamics change as a function of the behavior or state of the animal (Crochet and Petersen, 2006; Okun et al., 2010; Constantinople and Bruno, 2011). Intracellular recordings from cortical pyramidal cells have shown that active behavioral states are associated with a reduction of low-frequency voltage fluctuations (Crochet and Petersen, 2006; Poulet and Petersen, 2008; Constantinople and Bruno, 2011; Crochet et al., 2011). Consistent with this interpretation, there exists evidence that the relative SD values of $g_{i}$ and $g_{e}$ change as a function of the network state and sensory input (Rudolph et al., 2007; Wu et al., 2008). Hence, different network regimes associated with different synaptic weights and kinetics or neuronal gain could be used to control the relative size of $g_{i}$ and $g_{e}$ fluctuations and modulate the spectral properties of membrane voltage and spike output.

\section{References}

Adesnik H, Scanziani M (2010) Lateral competition for cortical space by layer-specific horizontal circuits. Nature 464:1155-1160. CrossRef Medline

Alonso A, García-Austt E (1987) Neuronal sources of theta rhythm in the entorhinal cortex of the rat. II. Phase relations between unit discharges and theta field potentials. Exp Brain Res 67:502-509. CrossRef Medline

Alonso A, Llinás RR (1989) Subthreshold Na+-dependent theta-like rhythmicity in stellate cells of entorhinal cortex layer II. Nature 342:175-177. CrossRef Medline

Anderson JS, Lampl I, Gillespie DC, Ferster D (2001) Membrane potential and conductance changes underlying length tuning of cells in cat primary visual cortex. J Neurosci 21:2104-2112. Medline

Atallah BV, Scanziani M (2009) Instantaneous modulation of gamma oscillation frequency by balancing excitation with inhibition. Neuron 62:566577. CrossRef Medline

Bettencourt JC, Lillis KP, Stupin LR, White JA (2008) Effects of imperfect dynamic clamp: computational and experimental results. J Neurosci Methods 169:282-289. CrossRef Medline

Brandon MP, Bogaard AR, Libby CP, Connerney MA, Gupta K, Hasselmo ME (2011) Reduction of theta rhythm dissociates grid cell spatial periodicity from directional tuning. Science 332:595-599. CrossRef Medline

Broicher T, Malerba P, Dorval AD, Borisyuk A, Fernandez FR, White JA (2012) Spike phase locking in CAl pyramidal neurons depends on background conductance and firing rate. J Neurosci 32:14374-14388. CrossRef Medline

Brunel N, Chance FS, Fourcaud N, Abbott LF (2001) Effects of synaptic noise and filtering on the frequency response of spiking neurons. Phys Rev Lett 86:2186-2189. CrossRef Medline

Burgalossi A, Herfst L, von Heimendahl M, Förste H, Haskic K, Schmidt M, Brecht M (2011) Microcircuits of functionally identified neurons in the rat medial entorhinal cortex. Neuron 70:773-786. CrossRef Medline

Burgess N, Barry C, O'Keefe J (2007) An oscillatory interference model of grid cell firing. Hippocampus 17:801-812. CrossRef Medline

Burton BG, Economo MN, Lee GJ, White JA (2008) Development of theta rhythmicity in entorhinal stellate cells of the juvenile rat. J Neurophysiol 100:3144-3157. CrossRef Medline

Buzsáki G, Draguhn A (2004) Neuronal oscillations in cortical networks. Science 304:1926-1929. CrossRef Medline

Cardin JA, Palmer LA, Contreras D (2008) Cellular mechanisms underlying stimulus-dependent gain modulation in primary visual cortex neurons in vivo. Neuron 59:150-160. CrossRef Medline

Constantinople CM, Bruno RM (2011) Effects and mechanisms of wakefulness on local cortical networks. Neuron 69:1061-1068. CrossRef Medline

Crochet S, Petersen CC (2006) Correlating whisker behavior with membrane potential in barrel cortex of awake mice. Nat Neurosci 9:608-610. CrossRef Medline

Crochet S, Poulet JF, Kremer Y, Petersen CC (2011) Synaptic mechanisms underlying sparse coding of active touch. Neuron 69:1160-1175. CrossRef Medline

Csicsvari J, Hirase H, Czurkó A, Mamiya A, Buzsáki G (1999) Oscillatory coupling of hippocampal pyramidal cells and interneurons in the behaving rat. J Neurosci 19:274-287. Medline

Destexhe A, Paré D (1999) Impact of network activity on the integrative properties of neocortical pyramidal neurons in vivo. J Neurophysiol 81: 1531-1547. Medline

Destexhe A, Rudolph M, Fellous JM, Sejnowski TJ (2001) Fluctuating synaptic conductances recreate in vivo-like activity in neocortical neurons. Neuroscience 107:13-24. CrossRef Medline

Economo MN, White JA (2012) Membrane properties and the balance between excitation and inhibition control gamma-frequency oscillations arising from feedback inhibition. PLoS Comput Biol 8:e1002354. CrossRef Medline

Epsztein J, Lee AK, Chorev E, Brecht M (2010) Impact of spikelets on hippocampal CA1 pyramidal cell activity during spatial exploration. Science 327:474-477. CrossRef Medline

Fellous JM, Rudolph M, Destexhe A, Sejnowski TJ (2003) Synaptic background noise controls the input/output characteristics of single cells in an in vitro model of in vivo activity. Neuroscience 122:811-829. CrossRef Medline

Fernandez FR, White JA (2008) Artificial synaptic conductances reduce subthreshold oscillations and periodic firing in stellate cells of the entorhinal cortex. J Neurosci 28:3790-3803. CrossRef Medline

Fernandez FR, White JA (2010) Gain control in CA1 pyramidal cells using changes in somatic conductance. J Neurosci 30:230-241. CrossRef Medline

Fernandez FR, Broicher T, Truong A, White JA (2011) Membrane voltage fluctuations reduce spike frequency adaptation and preserve output gain in CA1 pyramidal neurons in a high-conductance state. J Neurosci 31: 3880-3893. CrossRef Medline

Fourcaud-Trocmé N, Hansel D, van Vreeswijk C, Brunel N (2003) How spike generation mechanisms determine the neuronal response to fluctuating inputs. J Neurosci 23:11628-11640. Medline

Fox SE, Wolfson S, Ranck JB Jr (1986) Hippocampal theta rhythm and the firing of neurons in walking and urethane anesthetized rats. Exp Brain Res 62:495-508. Medline

Frank LM, Brown EN, Wilson MA (2001) A comparison of the firing properties of putative excitatory and inhibitory neurons from CAl and the entorhinal cortex. J Neurophysiol 86:2029-2040. Medline

Franks KM, Russo MJ, Sosulski DL, Mulligan AA, Siegelbaum SA, Axel R (2011) Recurrent circuitry dynamically shapes the activation of piriform cortex. Neuron 72:49-56. CrossRef Medline

Fries P, Reynolds JH, Rorie AE, Desimone R (2001) Modulation of oscillatory neuronal synchronization by selective visual attention. Science 291: 1560-1563. CrossRef Medline

Garden DL, Dodson PD, O'Donnell C, White MD, Nolan MF (2008) Tuning of synaptic integration in the medial entorhinal cortex to the organization of grid cell firing fields. Neuron 60:875-889. CrossRef Medline

Giocomo LM, Zilli EA, Fransén E, Hasselmo ME (2007) Temporal frequency of subthreshold oscillations scales with entorhinal grid cell field spacing. Science 315:1719-1722. CrossRef Medline

Giocomo LM, Hussaini SA, Zheng F, Kandel ER, Moser M-B, Moser MB (2011) Grid cells use HCN1 channels for spatial scaling. Cell 147:1159_ 1170. CrossRef Medline

Haas JS, White JA (2002) Frequency selectivity of layer II stellate cells in the medial entorhinal cortex. J Neurophysiol 88:2422-2429. CrossRef Medline

Haas JS, Dorval AD 2nd, White JA (2007) Contributions of Ih to feature selectivity in layer II stellate cells of the entorhinal cortex. J Comput Neurosci 22:161-171. CrossRef Medline

Hafting T, Fyhn M, Molden S, Moser MB, Moser EI (2005) Microstructure of a spatial map in the entorhinal cortex. Nature 436:801-806. CrossRef Medline

Harvey CD, Collman F, Dombeck DA, Tank DW (2009) Intracellular dynamics of hippocampal place cells during virtual navigation. Nature 461: 941-946. CrossRef Medline

Heiss JE, Katz Y, Ganmor E, Lampl I (2008) Shift in the balance between excitation and inhibition during sensory adaptation of S1 neurons. J Neurosci 28:13320-13330. CrossRef Medline

Heys JG, Giocomo LM, Hasselmo ME (2010) Cholinergic modulation of the resonance properties of stellate cells in layer II of medial entorhinal cortex. J Neurophysiol 104:258-270. CrossRef Medline 
Isaacson JS, Scanziani M (2011) How inhibition shapes cortical activity. Neuron 72:231-243. CrossRef Medline

Kispersky TJ, Fernandez FR, Economo MN, White JA (2012) Spike resonance properties in hippocampal O-LM cells are dependent on refractory dynamics. J Neurosci 32:3637-3651. CrossRef Medline

Klausberger T, Somogyi P (2008) Neuronal diversity and temporal dynamics: the unity of hippocampal circuit operations. Science 321:53-57. CrossRef Medline

Klausberger T, Magill PJ, Márton LF, Roberts JD, Cobden PM, Buzsáki G, Somogyi P (2003) Brain-state- and cell-type-specific firing of hippocampal interneurons in vivo. Nature 421:844-848. CrossRef Medline

Koenig J, Linder AN, Leutgeb JK, Leutgeb S (2011) The spatial periodicity of grid cells is not sustained during reduced theta oscillations. Science 332: 592-595. CrossRef Medline

Köndgen H, Geisler C, Fusi S, Wang XJ, Lüscher HR, Giugliano M (2008) The dynamical response properties of neocortical neurons to temporally modulated noisy inputs in vitro. Cereb Cortex 18:2086-2097. Medline

Liu BH, Li P, Sun YJ, Li YT, Zhang LI, Tao HW (2010) Intervening inhibition underlies simple-cell receptive field structure in visual cortex. Nat Neurosci 13:89-96. CrossRef Medline

Nolan MF, Dudman JT, Dodson PD, Santoro B (2007) HCN1 channels control resting and active integrative properties of stellate cells from layer II of the entorhinal cortex. J Neurosci 27:12440-12451. CrossRef Medline

O'Keefe J, Burgess N (2005) Dual phase and rate coding in hippocampal place cells: theoretical significance and relationship to entorhinal grid cells. Hippocampus 15:853-866. CrossRef Medline

O'Keefe J, Recce ML (1993) Phase relationship between hippocampal place units and the EEG theta rhythm. Hippocampus 3:317-330. CrossRef Medline

Okun M, Lampl I (2008) Instantaneous correlation of excitation and inhibition during ongoing and sensory-evoked activities. Nat Neurosci 11: 535-537. CrossRef Medline

Okun M, Naim A, Lampl I (2010) The subthreshold relation between cortical local field potential and neuronal firing unveiled by intracellular recordings in awake rats. J Neurosci 30:4440-4448. CrossRef Medline

Paré D, Shink E, Gaudreau H, Destexhe A, Lang EJ (1998) Impact of spontaneous synaptic activity on the resting properties of cat neocortical pyramidal neurons in vivo. J Neurophysiol 79:1450-1460. Medline

Pécseli HL (2000) Fluctuations in physical systems. Cambridge, UK: Cambridge UP.

Pouille F, Scanziani M (2001) Enforcement of temporal fidelity in pyramidal cells by somatic feed-forward inhibition. Science 293:1159-1163. CrossRef Medline

Poulet JF, Petersen CC (2008) Internal brain state regulates membrane potential synchrony in barrel cortex of behaving mice. Nature 454:881-885. CrossRef Medline
Quilichini P, Sirota A, Buzsáki G (2010) Intrinsic circuit organization and theta-gamma oscillation dynamics in the entorhinal cortex of the rat. J Neurosci 30:11128-11142. CrossRef Medline

Renart A, de la Rocha J, Bartho P, Hollender L, Parga N, Reyes A, Harris KD (2010) The asynchronous state in cortical circuits. Science 327:587-590. CrossRef Medline

Richardson MJ, Gerstner W (2005) Synaptic shot noise and conductance fluctuations affect the membrane voltage with equal significance. Neural Comput 17:923-947. CrossRef Medline

Richardson MJ, Brunel N, Hakim V (2003) From subthreshold to firingrate resonance. J Neurophysiol 89:2538-2554. CrossRef Medline

Rudolph M, Pospischil M, Timofeev I, Destexhe A (2007) Inhibition determines membrane potential dynamics and controls action potential generation in awake and sleeping cat cortex. J Neurosci 27:5280-5290. CrossRef Medline

Rutishauser U, Ross IB, Mamelak AN, Schuman EM (2010) Human memory strength is predicted by theta-frequency phase-locking of single neurons. Nature 464:903-907. CrossRef Medline

Sceniak MP, Sabo SL (2010) Modulation of firing rate by background synaptic noise statistics in rat visual cortical neurons. J Neurophysiol 104: 2792-2805. CrossRef Medline

Schmidt-Hieber C, Häusser M (2013) Cellular mechanisms of spatial navigation in the medial entorhinal cortex. Nat Neurosci 16:325-331. CrossRef Medline

Shu Y, Hasenstaub A, McCormick DA (2003) Turning on and off recurrent balanced cortical activity. Nature 423:288-293. CrossRef Medline

Stewart M, Quirk GJ, Barry M, Fox SE (1992) Firing relations of medial entorhinal neurons to the hippocampal theta rhythm in urethane anesthetized and walking rats. Exp Brain Res 90:21-28. Medline

Suzuki N, Bekkers JM (2012) Microcircuits mediating feedforward and feedback synaptic inhibition in the piriform cortex. J Neurosci 32:919931. CrossRef Medline

Vogels TP, Abbott LF (2009) Gating multiple signals through detailed balance of excitation and inhibition in spiking networks. Nat Neurosci 12: 483-491. CrossRef Medline

Waters J, Helmchen F (2006) Background synaptic activity is sparse in neocortex. J Neurosci 26:8267-8277. CrossRef Medline

Wehr M, Zador AM (2003) Balanced inhibition underlies tuning and sharpens spike timing in auditory cortex. Nature 426:442-446. CrossRef Medline

Wei W, Wolf F (2011) Spike onset dynamics and response speed in neuronal populations. Phys Rev Lett 106:088102. CrossRef Medline

Wu GK, Arbuckle R, Liu BH, Tao HW, Zhang LI (2008) Lateral sharpening of cortical frequency tuning by approximately balanced inhibition. Neuron 58:132-143. CrossRef Medline 